\title{
PTF10nvg: AN OUTBURSTING CLASS I PROTOSTAR IN THE PELICAN/NORTH AMERICAN NEBULA
}

\author{
Kevin R. Covey ${ }^{1,11,12}$, Lynne A. Hillennrand ${ }^{2}$, Adam A. Miller ${ }^{3}$, Dovi Poznanski ${ }^{3,4,14}$, S. Bradley Cenko ${ }^{3}$, \\ Jeffrey M. Silverman ${ }^{3}$, Joshua S. Bloom ${ }^{3}$, Mansi M. Kasliwal ${ }^{2}$, William Fischer ${ }^{5}$, John Rayner ${ }^{6,13}$, Luisa M. Rebull ${ }^{7}$, \\ Nathaniel R. Butler ${ }^{3,14}$, Alexei V. Filippenko ${ }^{3}$, Nicholas M. LaW ${ }^{8}$, Eran O. Ofek ${ }^{2,14}$, Marcel Agüeros ${ }^{9}$, \\ Richard G. Dekany ${ }^{10}$, Gustavo Rahmer ${ }^{10}$, David Hale ${ }^{10}$, Roger Smith ${ }^{10}$, Robert M. Quimby ${ }^{2}$, Peter Nugent ${ }^{4}$, \\ Janet Jacobsen $^{4}$, JefF Zolkower ${ }^{10}$, Viswa Velur ${ }^{10}$, Richard Walters ${ }^{10}$, John Henning $^{10}$, Khanh Bui $^{10}$, Dan McKenna ${ }^{10}$, \\ Shrinivas R. KULKARNI ${ }^{2}$, AND Christopher KLEIN ${ }^{3}$ \\ ${ }^{1}$ Department of Astronomy, Cornell University, 226 Space Sciences Building, Ithaca, NY 14853, USA \\ 2 Astrophysics Department, California Institute of Technology, Pasadena, CA 91125, USA \\ ${ }^{3}$ Department of Astronomy, University of California, Berkeley, CA 94720-3411, USA \\ ${ }^{4}$ Computational Cosmology Center, Lawrence Berkeley National Laboratory, 1 Cyclotron Road, Berkeley, CA 94720, USA \\ ${ }^{5}$ Department of Physics and Astronomy, University of Toledo, 2801 West Bancroft Street, Toledo, OH 43606, USA \\ ${ }^{6}$ Institute for Astronomy, University of Hawai, 2680 Woodlawn Drive, Honolulu, HI 96822, USA \\ ${ }^{7}$ Spitzer Science Center, California Institute of Technology, Pasadena, CA 91125, USA \\ ${ }^{8}$ Dunlap Institute for Astronomy and Astrophysics, University of Toronto, 50 St. George Street, Toronto M5S 3H4, Ontario, Canada \\ ${ }^{9}$ Columbia Astrophysics Laboratory, Columbia University, New York, NY 10027, USA \\ ${ }^{10}$ Caltech Optical Observatories, California Institute of Technology, Pasadena, CA 91125, USA \\ Received 2010 October 14; accepted 2010 November 12; published 2011 January 5
}

\begin{abstract}
During a synoptic survey of the North American Nebula region, the Palomar Transient Factory (PTF) detected an optical outburst (dubbed PTF10nvg) associated with the previously unstudied flat or rising spectrum infrared source IRAS 20496+4354. The PTF $R$-band light curve reveals that PTF10nvg brightened by more than 5 mag during the current outburst, rising to a peak magnitude of $R_{\mathrm{PTF}} \approx 13.5$ in 2010 September. Follow-up observations indicate that PTF10nvg has undergone a similar $\sim 5$ mag brightening in the $K$ band and possesses a rich emissionline spectrum, including numerous lines commonly assumed to trace mass accretion and outflows. Many of these lines are blueshifted by $\sim 175 \mathrm{~km} \mathrm{~s}^{-1}$ from the North American Nebula's rest velocity, suggesting that PTF10nvg is driving an outflow. Optical spectra of PTF10nvg show several TiO/VO band heads fully in emission, indicating the presence of an unusual amount of dense $\left(>10^{10} \mathrm{~cm}^{-3}\right)$, warm $(1500-4000 \mathrm{~K})$ circumstellar material. Nearinfrared spectra of PTF10nvg appear quite similar to a spectrum of McNeil's Nebula/V1647 Ori, a young star which has undergone several brightenings in recent decades, and $06297+1021 \mathrm{~W}$, a Class I protostar with a similarly reached near-infrared emission line spectrum. While further monitoring is required to fully understand this event, we conclude that the brightening of PTF10nvg is indicative of enhanced accretion and outflow in this Class-I-type protostellar object, similar to the behavior of V1647 Ori in 2004-2005.
\end{abstract}

Key words: stars: emission-line, $\mathrm{Be}$ - stars: formation - stars: pre-main sequence - stars: variables: general stars: winds, outflows

Online-only material: color figures

\section{INTRODUCTION}

A defining characteristic (e.g., Joy 1945) of young stars is their photometric variability. At optical wavelengths, periodic low-amplitude variations are thought to arise from surface inhomogeneities on a rotating star; aperiodic large-amplitude variability is more often attributed to accretion-related activity. The "activity" amplitude generally declines as the star ages and the disk evolves, with "outburst" behavior exhibited by some of the most active sources. This has resulted in the definition of EXor type (actually named after EX Lup) young-star variables; these objects often undergo repeated outbursts with 2-3 mag amplitudes and characteristic timescales of weeks to months (Herbig et al. 2001; Herbig 2008; Lorenzetti et al. 2009).

\footnotetext{
${ }^{11}$ Hubble Fellow.

12 Visiting Researcher, Department of Astronomy, Boston University, 725 Commonwealth Avenue, Boston, MA 02215, USA.

${ }^{13}$ Visiting Astronomer at the Infrared Telescope Facility, which is operated by the University of Hawaii under Cooperative Agreement no. NNX-08AE38A with the National Aeronautics and Space Administration (NASA), Science Mission Directorate, Planetary Astronomy Program.

${ }^{14}$ Einstein Fellow.
}

More extreme are the FU Ori-type variables, which undergo 4-6 mag amplitude increases over a few months, followed by a slow, decades-long decay (Herbig 1989; Hartmann \& Kenyon 1996). In both cases, the variability is commonly attributed to nonsteady mass accretion from a circumstellar disk. Theoretical models typically invoke thermal, gravitational, or magnetorotational disk instabilities to explain these accretion outbursts (Bell \& Lin 1994; Kley \& Lin 1999; Armitage et al. 2001; Vorobyov \& Basu 2005; Boley et al. 2006; Zhu et al. 2009), potentially triggered by binary interactions (Reipurth \& Aspin 2004b; Lodato \& Clarke 2004). Recently documented outbursting young stars include V1647 Ori (McNeil et al. 2004; Briceño et al. 2004; Reipurth \& Aspin 2004a; Walter et al. 2004; Ojha et al. 2006; Fedele et al. 2007; Aspin et al. 2009), V1118 Ori (Lorenzetti et al. 2007; Audard et al. 2010), V733 Cep (Reipurth et al. 2007; Peneva et al. 2010), ZCMa (Szeifert et al. 2010; Whelan et al. 2010), and EX Lup itself (Herbig et al. 2001).

With optical and near-infrared telescopes now regularly patrolling the sky, and ongoing variability surveys that target active star-forming regions, the full gamut of young-star variability will soon be revealed, quantifying the characteristic timescales 

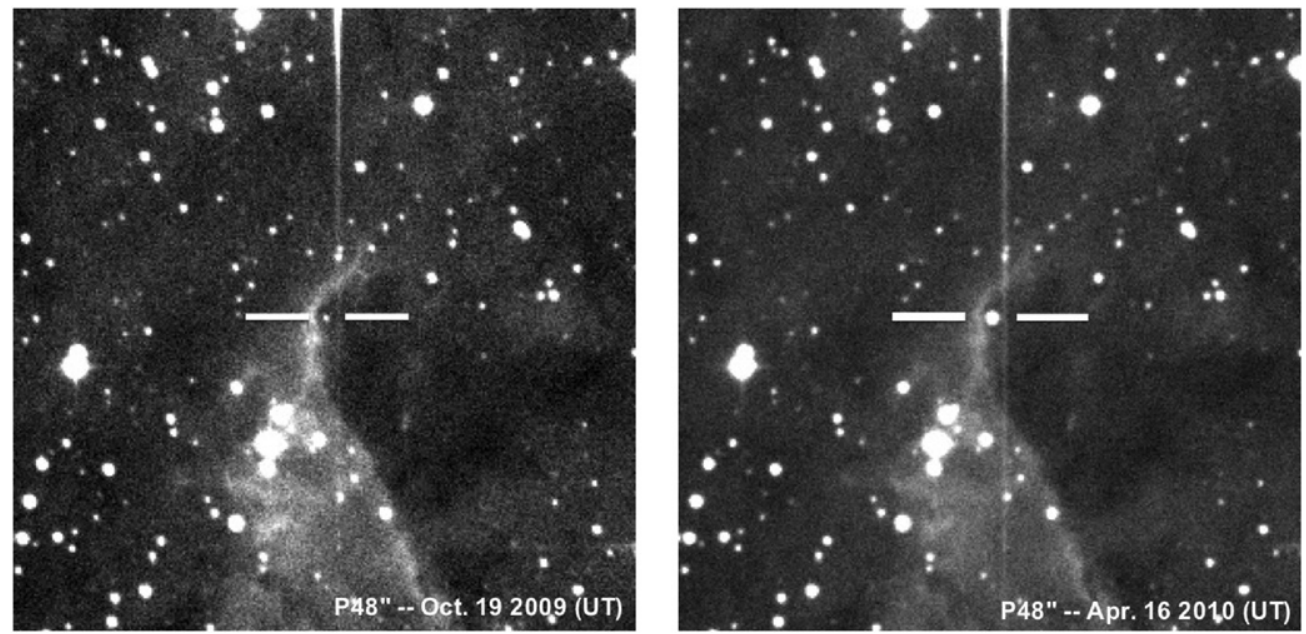

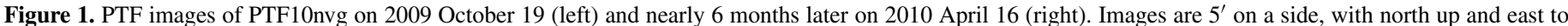

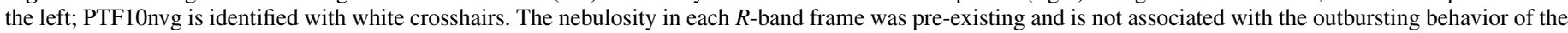

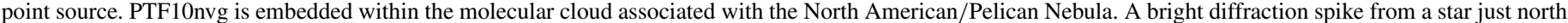
of PTF10nvg does not substantially affect the PSF photometry derived for PTF10nvg.

for eruptive events of varying amplitudes. One such ongoing investigation is using the Palomar 48 inch Schmidt telescope and the Palomar Transient Factory (PTF; Law et al. 2009; Rau et al. 2009) infrastructure to catalogue photometric variability in the North American/Pelican Nebula region of recent star formation. Located at a distance of $\sim 600 \mathrm{pc}$, the population of young stars in this region (identified largely from surveys in $\mathrm{H} \alpha$; e.g., Herbig 1958; Welin 1973; Ogura et al. 2002, or the mid-IR, e.g., Guieu et al. 2009; L. M. Rebull et al. 2011, in preparation) is not as well studied as members of more proximate star-forming regions, but already includes a disproportionately large number of the known members of the exclusive FU Ori class, namely V1057 Cyg and V1515 Cyg. These two objects, along with FU Ori itself, are the defining members of the class; they exhibit strong wind signatures in lines such as $\mathrm{Na}$ I $\mathrm{D}$ and $\mathrm{H} \alpha$, metallic absorption patterns similar to those of FG-type supergiants in the optical and M-type supergiants in the IR, and large thermal IR luminosity.

We have identified a new outbursting object located at position $\alpha=20^{\mathrm{h}} 51^{\mathrm{m}} 26.23, \delta=+44^{\circ} 05^{\prime} 23^{\prime \prime} .9$ (J2000.0), just south of the Pelican Nebula. It sits within the apex of a nebular arc that wraps around the object from northwest to the east and then to the south. The source is located within $1^{\prime \prime}$ of the position of IRAS $20496+4354$, well inside the $16^{\prime \prime}$ error ellipse associated with that detection. The source was further detected by the Midcourse Space Experiment (MSX) in 1996-1997, Spitzer in 2006 August 9-11 (Guieu et al. 2009; L. M. Rebull et al. 2011, in preparation), and AKARI/IRC during its 2006-2007 mission.

At shorter wavelengths, the source was not visible $(K>$ $15.3 \mathrm{mag}$ ) to the Two Micron All Sky Survey (2MASS; Skrutskie et al. 2006). The 2-24 $\mu \mathrm{m}$ spectral index $(\alpha=d$ $\log \left(\lambda F_{\lambda}\right) d \log \lambda$; Lada 1987) implied by the 2 MASS $K$-band upper limit and the IRAS $24 \mu \mathrm{m}$ detection is $\alpha \gtrsim 1.7$, consistent with a designation as a heavily embedded Class I protostar in the classification system devised by Lada (1987). The source does, however, appear in the $J$ and $K$ images of Magnier et al. (1999) that were obtained as follow-up observations to the IRAS detection. The source is not obvious on Digital Sky Survey (DSS) images (implying $m_{\mathrm{pg}}>21 \mathrm{mag}$ ), but NOMAD, using DSS-2 data, reports $m_{B}=20.17$ and $m_{R}=18.28 \mathrm{mag}$ for a source 3 .' 4 away from the source position reported here (Zacharias et al. 2005). Images of the field taken with $\mathrm{H} \alpha$ and
[S II] filters by Bally \& Reipurth (2003) appear to show faint smudges at the source position.

We report here the outburst discovery data, as well as followup multi-filter optical and near-infrared (NIR) photometric and spectroscopic monitoring that was obtained during various stages of the 2010 outburst. We find evidence for strong emission lines and broadly absorbed wind features, similar to other outbursting young objects, but also some unique spectroscopic attributes, including molecular emission from $\mathrm{TiO}$ and $\mathrm{VO}$. We analyze the data in the context of an eruptive Class-I type, heavily embedded protostar.

\section{OBSERVATIONS}

\subsection{Optical Photometry}

During the 2009 and 2010 observing seasons, PTF obtained red optical images of the North American/Pelican Nebula star-forming region with a typical five-day cadence. These observations were conducted with the main PTF Survey Camera, the former CFHT12K mosaic camera now extensively reengineered and mounted on the 48 inch Samuel Oschin telescope at Palomar Observatory (hereafter "P48"). The camera is a mosaic of 11 CCDs, covering a $7.8 \mathrm{deg}^{2}$ field of view with $1^{\prime \prime}$ sampling; typical conditions at Palomar Observatory produce 2 ". 0 full width at half-maximum (FWHM) intensity images (Law et al. 2009). The $R_{\mathrm{PTF}}$ filter, a Mould $R$ band, is similar to SDSS- $r$ in shape but is shifted redward by $\sim 27 \AA$ and is $\sim 20 \AA$ wider. The typical $5 \sigma$ limiting magnitudes are $m_{R} \approx 20.6(\mathrm{AB})$ in $60 \mathrm{~s}$ exposures. Representative images of PTF10nvg from the 2009 and 2010 observing seasons are shown in Figure 1.

Transient sources are detected in PTF monitoring data by means of automated reduction pipelines, including a near-realtime image-subtraction pipeline hosted at Lawrence Berkeley National Laboratory (LBNL). Well-detected sources in the difference images are scored (using a human trained machinebased classifier) for their likelihood of being truly astrophysical in nature or of spurious origin. Variable sources with larger likelihoods of being nonspurious are passed to an automatic source classifier at UC Berkeley ("Oarical"), which combines PTF measurements with all other available information (e.g., SIMBAD identifications, 2MASS photometry, etc.) to provide probabilistic classifications of PTF detections (J. S. Bloom 

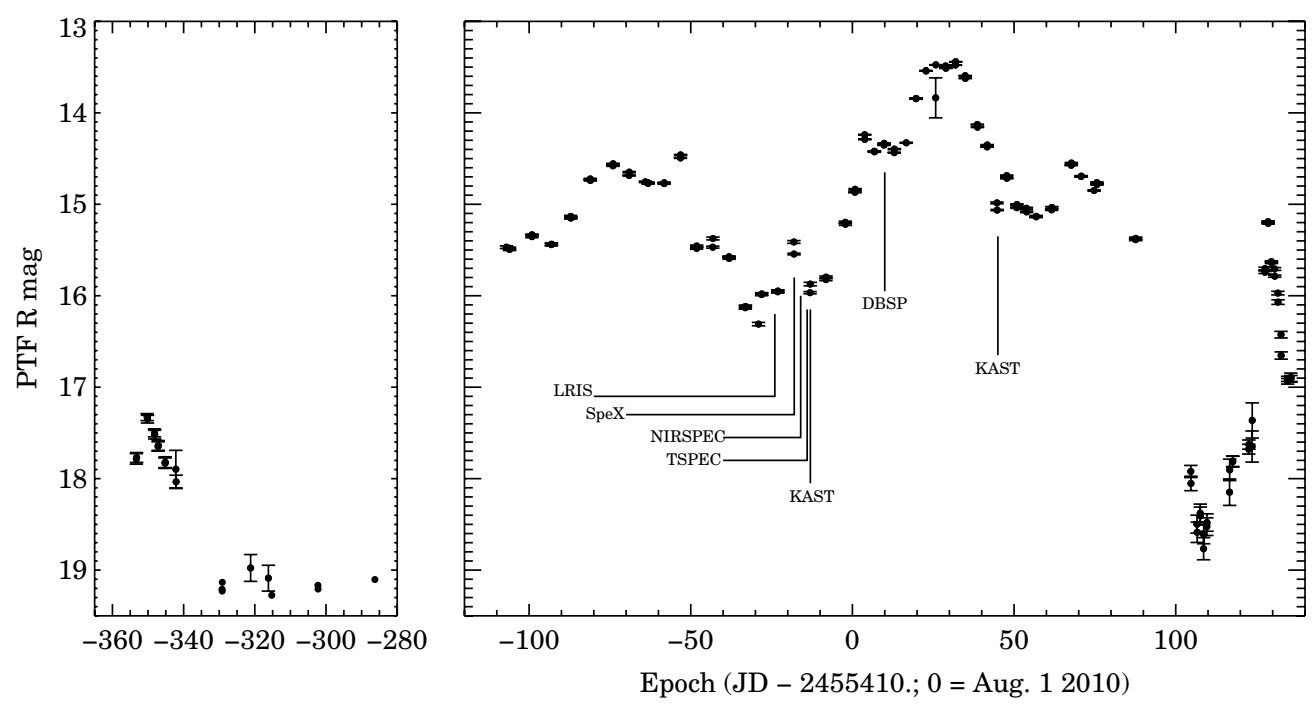

Figure 2. $R_{\mathrm{PTF}}$-band light curve of PTF10nvg, measured via PSF photometry of individual P48 frames (see Section 2.1 for details). Left panel shows observations from the 2009 season; right panel shows 2010 observations, with labels identifying the timing of the spectra presented in Sections 2.3 and 2.4

et al. 2011, in preparation). These initial classifications are made available to PTF collaboration members via the PTF Follow-up Marshal, which enables visual inspection of current and reference images, precursor PTF light curves, and any subsequent PTF spectroscopy.

The source reported here was detected by the PTF pipeline and automatically assigned the name 10nvg, following PTF naming conventions. PTF images are typically re-reduced for sources of particular interest, such as PTF10nvg, via a "whiteglove pipeline," where PSF photometry is performed on individual frames. The PSF photometry is calculated using a modified version of the pipeline developed by the Supernova Legacy Survey (see Astier et al. 2006). Absolute calibration is performed relative to the USNO-B1 catalog (Monet et al. 2003), and typically has an uncertainty of $\sim 0.15 \mathrm{mag}$. The full P48 light curve of PTF10nvg produced by this "white-glove reduction" is shown in Figure 2 and the photometry appears in Table 1.

The PTF also makes use of the robotic Palomar 60 inch telescope (P60; Cenko et al. 2006) to obtain multi-color photometry for source verification and classification purposes. PTF10nvg was observed by the robotic Palomar 60-inch starting 2010 August 1.35 in the $r$-band, $i$-band, and $z$-band filters. The absolute zero-point calibration was derived relative to the Sloan Digital Sky Survey (SDSS) from separate fields observed by the Palomar 60 inch during the night of 2010 September 3 with the same filter. All other fields are calibrated relative to this night. Figure 3 presents a three-color image constructed from the P60 riz frames, and Table 2 contains the individual measurements.

We note that there is a $\sim 0.45$ mag offset between the P48 $R_{\text {PTF }}$ and P60 $r$-band light curves for PTF10nvg. A simple zero-point error may contribute to this offset, but it is likely dominated by differences in each telescope's filter + detector response, and the different photometric systems underlying their calibration (Vega $R$ versus AB magnitude $r$ ). Given the multiple possible contributions to this offset, we have chosen to preserve each light curve on its native system, rather than explicitly transform them onto a common system.

\subsection{Near-infrared Photometry}

Near-infrared observations of PTF10nvg were conducted with the $1.3 \mathrm{~m}$ Peters Automated Infrared Imaging Telescope (PAIRITEL; Bloom et al. 2006) starting on 2010 July 10.

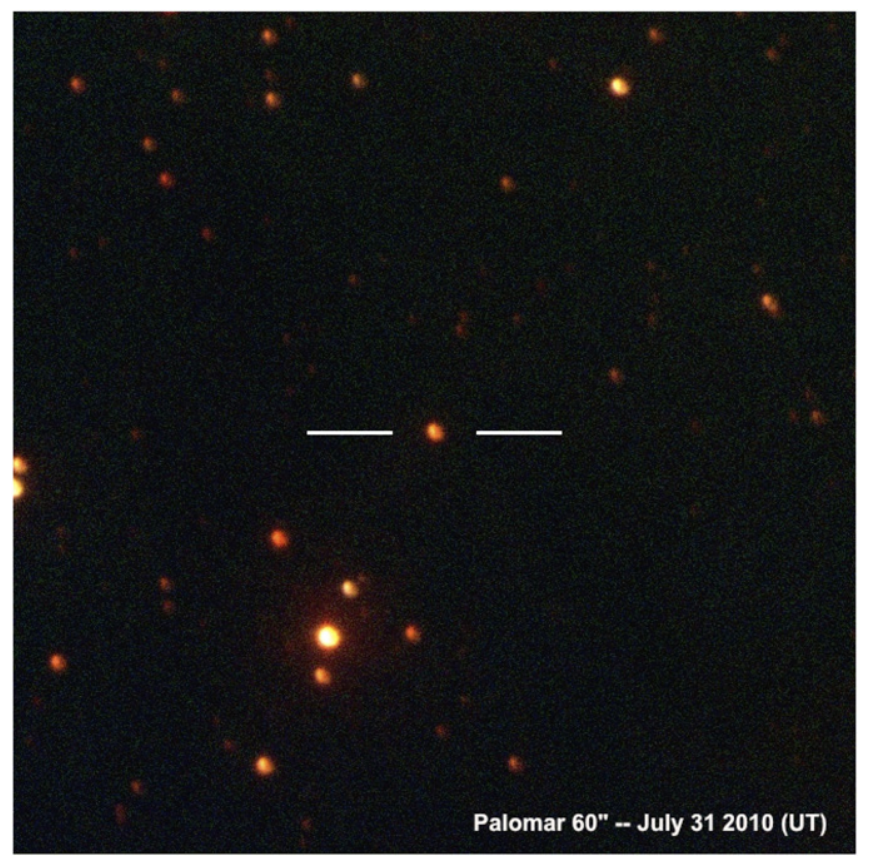

Figure 3. Three-color frame of PTF10nvg riz images obtained with the P60. The frame is 4.1 on a side, with north up and east to the left.

(A color version of this figure is available in the online journal.)

PAIRITEL is a roboticized system using the former 2MASS southern hemisphere survey camera that employs two dichroics to observe simultaneously in the $J, H$, and $K_{s}$ bands. Observations were scheduled and executed via a robotic system. PAIRITEL is operated in a fixed observing mode in which $7.8 \mathrm{~s}$ double-correlated images are created from the difference of a $7.851 \mathrm{~s}$ and a $51 \mathrm{~ms}$ integration taken in rapid succession (see Blake et al. 2008). The standard observing procedure involves taking three image pairs prior to dithering the telescope.

The raw data from these images are reduced using standard IR reduction methods via PAIRITEL PIPELINE III and the flux for all sources is measured via aperture photometry using SExtractor (Bertin \& Arnouts 1996), calibrated against 2MASS. PTF10nvg saturates the $7.851 \mathrm{~s}$ frames; however, PIPELINE III produces "short-frame" mosaics consisting of reduced, stacked 
Table 1

P48 R-Band Photometry of PTF10nvg

\begin{tabular}{|c|c|c|}
\hline $\begin{array}{c}\text { Epoch (JD } \\
-2,400,000)\end{array}$ & mag & $\begin{array}{c}1 \sigma \\
\text { Error }\end{array}$ \\
\hline 55056.7205 & 17.784 & 0.058 \\
\hline 55056.7954 & 17.768 & 0.054 \\
\hline 55059.7615 & 17.327 & 0.038 \\
\hline 55059.8570 & 17.349 & 0.042 \\
\hline 55061.8156 & 17.499 & 0.043 \\
\hline 55061.8834 & 17.519 & 0.048 \\
\hline 55062.8266 & 17.648 & 0.051 \\
\hline 55062.8906 & 17.637 & 0.053 \\
\hline 55064.7860 & 17.831 & 0.058 \\
\hline 55064.8311 & 17.820 & 0.059 \\
\hline 55067.8178 & 17.897 & 0.206 \\
\hline 55067.8929 & 18.035 & 0.073 \\
\hline 55080.7902 & 19.209 & 99.000 \\
\hline 55080.8339 & 19.229 & 99.000 \\
\hline 55080.8608 & 19.133 & 99.000 \\
\hline 55088.8156 & 18.976 & 0.147 \\
\hline 55093.8177 & 19.088 & 0.141 \\
\hline 55094.7525 & 19.275 & 99.000 \\
\hline 55107.7216 & 19.166 & 99.000 \\
\hline 55107.7656 & 19.207 & 99.000 \\
\hline 55123.7247 & 19.103 & 99.000 \\
\hline 55302.9792 & 15.469 & 0.014 \\
\hline 55303.9110 & 15.481 & 0.013 \\
\hline 55303.9593 & 15.494 & 0.011 \\
\hline 55310.8700 & 15.354 & 0.012 \\
\hline 55310.9130 & 15.334 & 0.008 \\
\hline 55316.8754 & 15.441 & 0.013 \\
\hline 55316.9198 & 15.437 & 0.012 \\
\hline 55322.8590 & 15.132 & 0.011 \\
\hline 55322.9028 & 15.151 & 0.010 \\
\hline 55328.9085 & 14.723 & 0.007 \\
\hline 55328.9530 & 14.739 & 0.006 \\
\hline 55335.9332 & 14.579 & 0.006 \\
\hline 55335.9774 & 14.557 & 0.006 \\
\hline 55340.9292 & 14.685 & 0.006 \\
\hline 55340.9735 & 14.648 & 0.007 \\
\hline 55345.9696 & 14.754 & 0.008 \\
\hline 55346.7699 & 14.765 & 0.007 \\
\hline 55346.8139 & 14.771 & 0.007 \\
\hline 55351.7712 & 14.774 & 0.008 \\
\hline 55351.8145 & 14.764 & 0.007 \\
\hline 55356.8089 & 14.493 & 0.005 \\
\hline 55356.8533 & 14.460 & 0.005 \\
\hline 55361.8110 & 15.482 & 0.009 \\
\hline 55361.8546 & 15.456 & 0.010 \\
\hline 55366.8177 & 15.469 & 0.011 \\
\hline 55366.8615 & 15.374 & 0.015 \\
\hline 55371.8399 & 15.573 & 0.009 \\
\hline 55371.8836 & 15.592 & 0.009 \\
\hline 55376.8771 & 16.131 & 0.016 \\
\hline 55376.9201 & 16.117 & 0.015 \\
\hline 55380.9668 & 16.310 & 0.019 \\
\hline 55381.9049 & 15.987 & 0.013 \\
\hline 55381.9551 & 15.980 & 0.013 \\
\hline 55386.8972 & 15.956 & 0.013 \\
\hline 55386.9442 & 15.949 & 0.013 \\
\hline 55391.8996 & 15.412 & 0.015 \\
\hline 55391.9437 & 15.544 & 0.009 \\
\hline 55396.9115 & 15.966 & 0.012 \\
\hline 55396.9655 & 15.873 & 0.019 \\
\hline 55401.8040 & 15.824 & 0.011 \\
\hline 55401.8483 & 15.796 & 0.012 \\
\hline 55407.7945 & 15.197 & 0.013 \\
\hline 55407.8421 & 15.218 & 0.012 \\
\hline 55410.7952 & 14.868 & 0.005 \\
\hline
\end{tabular}

Table 1

(Continued)

\begin{tabular}{|c|c|c|}
\hline $\begin{array}{c}\text { Epoch (JD } \\
-2,400,000)\end{array}$ & mag & $\begin{array}{c}1 \sigma \\
\text { Error }\end{array}$ \\
\hline 55410.8404 & 14.836 & 0.008 \\
\hline 55413.7918 & 14.239 & 0.004 \\
\hline 55413.8356 & 14.290 & 0.004 \\
\hline 55416.7871 & 14.420 & 0.005 \\
\hline 55416.8303 & 14.425 & 0.005 \\
\hline 55419.7818 & 14.352 & 0.004 \\
\hline 55419.8261 & 14.334 & 0.005 \\
\hline 55422.9468 & 14.435 & 0.005 \\
\hline 55422.9910 & 14.396 & 0.005 \\
\hline 55426.6605 & 14.327 & 0.005 \\
\hline 55429.6767 & 13.845 & 0.002 \\
\hline 55429.7202 & 13.842 & 0.003 \\
\hline 55432.7443 & 13.539 & 0.002 \\
\hline 55432.7874 & 13.542 & 0.002 \\
\hline 55435.7587 & 13.836 & 0.219 \\
\hline 55435.8028 & 13.475 & 0.002 \\
\hline 55438.8303 & 13.487 & 0.002 \\
\hline 55438.9133 & 13.512 & 0.002 \\
\hline 55441.8654 & 13.478 & 0.002 \\
\hline 55441.9300 & 13.441 & 0.002 \\
\hline 55444.8899 & 13.594 & 0.002 \\
\hline 55444.9340 & 13.623 & 0.002 \\
\hline 55448.6442 & 14.128 & 0.004 \\
\hline 55448.6889 & 14.157 & 0.004 \\
\hline 55451.6560 & 14.372 & 0.004 \\
\hline 55451.7002 & 14.351 & 0.004 \\
\hline 55454.7211 & 14.985 & 0.010 \\
\hline 55454.7651 & 15.063 & 0.007 \\
\hline 55457.7168 & 14.687 & 0.007 \\
\hline 55457.7608 & 14.715 & 0.006 \\
\hline 55460.8389 & 15.037 & 0.007 \\
\hline 55460.8875 & 15.002 & 0.007 \\
\hline 55463.8407 & 15.043 & 0.010 \\
\hline 55463.8859 & 15.083 & 0.008 \\
\hline 55466.8255 & 15.137 & 0.008 \\
\hline 55466.8771 & 15.132 & 0.009 \\
\hline 55471.6094 & 15.060 & 0.007 \\
\hline 55471.6884 & 15.035 & 0.010 \\
\hline 55477.6909 & 14.573 & 0.004 \\
\hline 55477.7342 & 14.549 & 0.006 \\
\hline 55480.7401 & 14.696 & 0.007 \\
\hline 55480.7835 & 14.692 & 0.006 \\
\hline 55484.7627 & 14.849 & 0.008 \\
\hline 55485.6193 & 14.763 & 0.007 \\
\hline 55485.6635 & 14.784 & 0.006 \\
\hline 55497.5978 & 15.386 & 0.010 \\
\hline 55497.6412 & 15.372 & 0.013 \\
\hline 55514.6697 & 17.921 & 0.066 \\
\hline 55514.7179 & 18.053 & 0.079 \\
\hline 55516.6017 & 18.498 & 0.098 \\
\hline 55516.6451 & 18.587 & 0.111 \\
\hline 55517.5770 & 18.378 & 0.100 \\
\hline 55517.6203 & 18.407 & 0.097 \\
\hline 55518.6247 & 18.766 & 0.121 \\
\hline 55518.6680 & 18.607 & 0.105 \\
\hline 55519.6221 & 18.525 & 0.097 \\
\hline 55519.6656 & 18.480 & 0.095 \\
\hline 55526.6565 & 18.148 & 0.145 \\
\hline 55526.7003 & 17.904 & 0.117 \\
\hline 55527.6085 & 17.810 & 0.056 \\
\hline 55527.6530 & 17.813 & 0.058 \\
\hline 55532.5921 & 17.678 & 0.053 \\
\hline 55532.6356 & 17.627 & 0.050 \\
\hline 55533.6257 & 17.649 & 0.170 \\
\hline 55533.6690 & 17.363 & 0.192 \\
\hline
\end{tabular}


Table 1

(Continued)

\begin{tabular}{ccc}
\hline \hline $\begin{array}{c}\text { Epoch }(\mathrm{JD} \\
-2,400,000)\end{array}$ & mag & $\begin{array}{c}1 \sigma \\
\text { Error }\end{array}$ \\
\hline 55537.6163 & 15.743 & 0.014 \\
55537.6606 & 15.702 & 0.014 \\
55538.5897 & 15.190 & 0.009 \\
55538.6337 & 15.208 & 0.008 \\
55539.5941 & 15.627 & 0.009 \\
55539.6384 & 15.638 & 0.010 \\
55540.6025 & 15.707 & 0.015 \\
55540.6464 & 15.786 & 0.011 \\
55541.6075 & 15.971 & 0.019 \\
55541.6511 & 16.069 & 0.025 \\
55542.5817 & 16.426 & 0.036 \\
55542.6250 & 16.654 & 0.040 \\
55544.5990 & 16.909 & 0.028 \\
55544.6459 & 16.935 & 0.030 \\
55545.6112 & 16.892 & 0.046 \\
55545.6554 & 16.907 & 0.035 \\
\hline
\end{tabular}

$51 \mathrm{~ms}$ images (see also Bloom et al. 2009). The "short-frame" mosaics contain $>10$ bright 2 MASS stars which we use to properly calibrate photometric measurements of PTF10nvg in these images. PAIRITEL has a known systematic uncertainty of $\sim 0.02-0.03$ mag in each of the $J, H$, and $K_{s}$ bands (see also Blake et al. 2008; Perley et al. 2010), which, in the case of PTF10nvg, is larger than the statistical error in all three bands. Thus, we add a systematic error of $0.03 \mathrm{mag}$ in quadrature with the statistical uncertainty to determine the total uncertainty in each band. The $J H K_{s}$ images of PTF10nvg obtained by PAIRITEL on 2010 July 10 are shown as a three-color frame in Figure 4; all $J H K_{s}$ magnitudes measured for PTF10nvg by PAIRITEL are presented in Table 3.

We also examined the 2MASS data products to determine if PTF10nvg was detected during that survey. A query of the 2MASS source catalog via the GATOR interface does not identify a source within $10^{\prime \prime}$ of this position, and visual inspection of the 2MASS images confirm that the source was undetected in 2000 October, when the field was imaged. Analysis of the 2MASS frames provides $5 \sigma$ upper limits on the brightness of the source at the epoch of the 2MASS observations of $J>16.35, H>15.38$, and $K_{s}>14.80$; we include these upper limits in the $J H K_{s}$ photometry presented in Table 3.

\subsection{Optical Spectroscopy}

Moderate-resolution optical spectra of PTF10nvg were obtained on four occasions: 2010 July 8 with the Low Resolution Imaging Spectrometer (LRIS; Oke et al. 1995; McCarthy et al. 1998; Steidel et al. 2004) on the Keck-I 10 m telescope, 2010 July 19 and 2010 September 16 with the Kast double spectrograph (Miller \& Stone 1993) on the Shane $3 \mathrm{~m}$ telescope at Lick Observatory, and 2010 August 11 with the Double Spectrograph (DBSP; Oke \& Gunn 1982) on the Hale 5 m telescope at Palomar Observatory.

LRIS observations were made by J.S.B., A. Cucchiara, A. N. Morgan, and D. A. Perley with the D560 dichroic, which has a $50 \%$ transmission split at $\lambda \approx 5700 \AA$, and a $1^{\prime \prime}$.0 long slit. The blue side of the spectrograph was configured with the $600 / 4000$ grism, providing $\sim 4 \AA$ resolution over the 3010 $5600 \AA$ wavelength range. The red spectrograph utilized the $400 / 8500$ grating, providing $\sim 7 \AA$ resolution over
Table 2

P60 Optical Photometry of PTF10nvg

\begin{tabular}{|c|c|c|c|}
\hline $\begin{array}{c}\text { Epoch (JD } \\
-2,400,000) \\
\end{array}$ & $\begin{array}{l}\text { Filter } \\
\text { Filter } \\
\end{array}$ & $\begin{array}{l}\mathrm{mag} \\
\mathrm{mag}\end{array}$ & $\begin{array}{c}1 \sigma \\
\text { Error } \\
\end{array}$ \\
\hline 55409.353 & $r$ & 15.487 & 0.170 \\
\hline 55409.355 & $z$ & 13.274 & 0.254 \\
\hline 55410.177 & $z$ & 13.134 & 0.332 \\
\hline 55411.234 & $z$ & 13.096 & 0.189 \\
\hline 55412.206 & $i$ & 14.208 & 0.025 \\
\hline 55412.207 & $z$ & 12.830 & 0.225 \\
\hline 55413.326 & $r$ & 14.776 & 0.150 \\
\hline 55413.327 & $z$ & 12.772 & 0.258 \\
\hline 55414.255 & $z$ & 12.864 & 0.266 \\
\hline 55415.280 & $z$ & 12.908 & 0.230 \\
\hline 55416.279 & $i$ & 14.218 & 0.043 \\
\hline 55416.280 & $z$ & 12.935 & 0.196 \\
\hline 55417.244 & $r$ & 14.920 & 0.159 \\
\hline 55417.245 & $z$ & 12.859 & 0.285 \\
\hline 55418.377 & $z$ & 12.947 & 0.240 \\
\hline 55419.316 & $z$ & 12.856 & 0.215 \\
\hline 55420.184 & $i$ & 14.105 & 0.040 \\
\hline 55420.185 & $z$ & 12.795 & 0.238 \\
\hline 55421.267 & $r$ & 15.059 & 0.162 \\
\hline 55421.268 & $z$ & 13.026 & 0.267 \\
\hline 55423.183 & $z$ & 12.994 & 0.188 \\
\hline 55424.408 & $i$ & 14.241 & 0.034 \\
\hline 55424.410 & $z$ & 12.896 & 0.257 \\
\hline 55433.350 & $i$ & 13.360 & 0.113 \\
\hline 55433.351 & $r$ & 13.877 & 0.191 \\
\hline 55433.352 & $z$ & 12.661 & 0.238 \\
\hline 55434.286 & $z$ & 11.860 & 0.546 \\
\hline 55436.256 & $z$ & 12.001 & 0.226 \\
\hline 55438.220 & $i$ & 13.285 & 0.226 \\
\hline 55438.298 & $r$ & 13.969 & 0.121 \\
\hline 55438.299 & $z$ & 12.089 & 0.153 \\
\hline 55442.213 & $i$ & 13.246 & 0.002 \\
\hline 55442.214 & $r$ & 13.899 & 0.002 \\
\hline 55442.214 & $z$ & 12.598 & 0.001 \\
\hline 55443.218 & $i$ & 13.219 & 0.018 \\
\hline 55443.220 & $r$ & 13.798 & 0.160 \\
\hline 55443.225 & $z$ & 11.963 & 0.250 \\
\hline 55444.239 & $i$ & 13.402 & 0.029 \\
\hline 55444.240 & $r$ & 13.978 & 0.186 \\
\hline 55444.241 & $z$ & 12.172 & 0.233 \\
\hline 55445.261 & $i$ & 13.572 & 0.020 \\
\hline 55445.265 & $r$ & 14.176 & 0.181 \\
\hline 55445.277 & $z$ & 12.336 & 0.231 \\
\hline 55446.280 & $i$ & 13.587 & 0.020 \\
\hline 55446.281 & $r$ & 14.194 & 0.181 \\
\hline 55446.282 & $z$ & 12.311 & 0.230 \\
\hline 55447.236 & $i$ & 13.875 & 0.029 \\
\hline 55447.237 & $z$ & 12.576 & 0.219 \\
\hline 55448.164 & $z$ & 12.693 & 0.196 \\
\hline 55449.372 & $z$ & 12.746 & 0.137 \\
\hline 55450.161 & $r$ & 14.513 & 0.161 \\
\hline 55450.162 & $z$ & 12.537 & 0.282 \\
\hline 55451.166 & $r$ & 14.863 & 0.129 \\
\hline 55452.355 & $z$ & 13.002 & 0.225 \\
\hline 55458.340 & $r$ & 15.241 & 0.159 \\
\hline 55458.341 & $z$ & 13.158 & 0.309 \\
\hline 55459.311 & $z$ & 13.334 & 0.200 \\
\hline 55460.228 & $z$ & 13.455 & 0.079 \\
\hline 55461.197 & $z$ & 13.382 & 0.144 \\
\hline 55462.270 & $r$ & 15.534 & 0.181 \\
\hline 55462.271 & $z$ & 13.463 & 0.122 \\
\hline 55463.187 & $z$ & 13.463 & 0.116 \\
\hline 55469.183 & $r$ & 15.763 & 0.190 \\
\hline 55469.184 & $z$ & 13.611 & 0.179 \\
\hline 55470.362 & $z$ & 13.576 & 0.160 \\
\hline
\end{tabular}



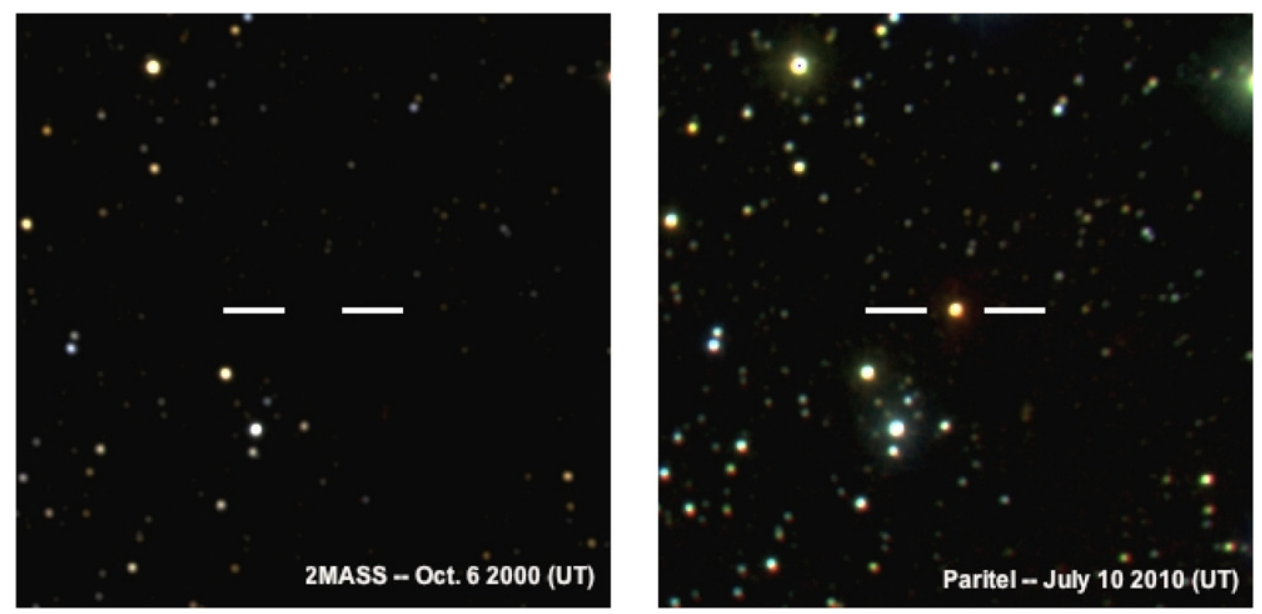

Figure 4. Three-color $J H K$ images of the field containing PTF10nvg obtained by 2MASS (left) and PAIRITEL (right). Frames are $5^{\prime}$ on a side, with north up and east to the left. PTF10nvg is not detected in the 2MASS imaging acquired on 2000 October 6 but is visible in the PAIRITEL imaging acquired on 2010 July 10. (A color version of this figure is available in the online journal.)

Table 2

(Continued)

\begin{tabular}{lccc}
\hline \hline $\begin{array}{c}\text { Epoch (JD } \\
-2,400,000)\end{array}$ & Filter & mag & $\begin{array}{c}1 \sigma \\
\text { Error }\end{array}$ \\
\hline 55473.308 & $r$ & 15.486 & 0.139 \\
55473.309 & $z$ & 13.421 & 0.138 \\
55477.322 & $r$ & 15.059 & 0.161 \\
55477.324 & $z$ & 13.042 & 0.166 \\
55478.146 & $z$ & 13.129 & 0.148 \\
55479.117 & $z$ & 13.047 & 0.075 \\
55480.171 & $z$ & 12.585 & 0.547 \\
55481.147 & $r$ & 15.335 & 0.089 \\
55481.148 & $z$ & 13.250 & 0.099 \\
55482.108 & $z$ & 13.317 & 0.160 \\
55483.128 & $z$ & 13.356 & 0.080 \\
55484.182 & $z$ & 13.360 & 0.179 \\
55485.109 & $r$ & 15.283 & 0.146 \\
55485.109 & $z$ & 13.321 & 0.177 \\
55496.280 & $r$ & 16.102 & 0.233 \\
55496.281 & $z$ & 14.078 & 0.228 \\
55497.130 & $z$ & 13.919 & 0.174 \\
55499.122 & $z$ & 13.969 & 0.103 \\
\hline & & &
\end{tabular}

Table 3

NIR Photometry of PTF10nvg

\begin{tabular}{crcrcrc}
\hline \hline Epoch (JD) & \multicolumn{1}{c}{$J$ mag } & $J$ Error & $H$ mag & $H$ Error & $K$ mag & $K$ Error \\
\hline 2451823.66652 & $>16.35$ & $\ldots$ & $>15.38$ & $\ldots$ & $>14.80$ & $\ldots$ \\
2455387.87633 & 12.22 & 0.07 & 10.26 & 0.02 & 8.45 & 0.01 \\
2455392.79441 & 12.23 & 0.01 & 10.38 & 0.06 & 8.45 & 0.02 \\
2455450.69076 & 10.88 & 0.02 & 9.39 & 0.01 & 8.06 & 0.01 \\
2455463.65116 & 11.48 & 0.03 & 9.86 & 0.01 & 8.39 & 0.01 \\
2455466.62568 & 11.62 & 0.03 & 10.02 & 0.01 & 8.38 & 0.01 \\
\hline
\end{tabular}

$5600-10300 \AA$. The LRIS spectra were reduced in the IRAF ${ }^{15}$ environment using standard routines. Cosmic rays were removed using the L.A. Cosmic routine (van Dokkum 2001). Spectra were extracted optimally (Horne 1986) and wavelength calibration was performed first relative to arc lamps and then tweaked based on night-sky lines in each individual image. Both air-to-vacuum and heliocentric corrections were then ap-

\footnotetext{
15 IRAF is distributed by the National Optical Astronomy Observatory, which is operated by the Association for Research in Astronomy, Inc., under cooperative agreement with the National Science Foundation.
}

plied to all spectra. Extracted spectra were divided through by a smoothed flux standard to remove narrow-band $(<50 \AA)$ instrumental effects (Bessell 1999). Finally, telluric absorption features were removed using the continuum from spectrophotometric standards (Wade \& Horne 1988; Matheson et al. 2000).

The July 19 Kast observations were obtained by C. V. Griffith and M. T. Kandrashoff, with reductions performed by J.M.S.; the September 16 observations were obtained by S.B.C. and M. T. Kandrashoff, with reductions performed by S.B.C. Kast was configured with a 2 " slit, a 600/4310 grism on the blue side, and a 300/7500 grating on the red side, yielding resolutions of $\sim 4$ and $\sim 10 \AA$, respectively.

DBSP data were acquired by L.M.R. using the D55 dichroic to obtain spectra with a 600 line $\mathrm{mm}^{-1}$ grating blazed at $4000 \AA$ from the atmospheric cutoff to $5500 \AA$ at $1.1 \AA$ resolution, and with a 158 line $\mathrm{mm}^{-1}$ grating blazed at $7500 \AA$ over the range 6300-8800 $\AA$ at $2.5 \AA$ dispersion. DBSP data reduction was performed by L.A.H., including flat fielding, extraction, and wavelength calibration, using standard routines in the IRAF environment.

\subsection{Near-infrared Spectroscopy}

Moderate-resolution NIR spectra of PTF10nvg were obtained on 2010 July 14 with the SpeX spectrograph (Rayner et al. 2003) at the NASA Infrared Telescope Facility, and on 2010 July 18 with the TripleSpec Spectrograph (Wilson et al. 2004) at the Apache Point Observatory.

SpeX observations were obtained by J.R. on 2010 July 14 with a 0.3 slit under clear skies and good seeing (0!4-0!'5). Both the short-cross-dispersed (SXD) and long cross-dispersed (LXD) modes were utilized, providing nearly contiguous coverage over the range $0.8-2.5 \mu \mathrm{m}$ with a spectral resolution of $R=2000$, and over 3-4.2 $\mu \mathrm{m}$ ( $L$ band) and 4.5$5.0 \mu \mathrm{m}$ ( $M$ band) at $R \approx 2500$. PTF10nvg was nodded along the slit during the observation, such that differencing consecutive images effectively removes the sky emission. The spectra were differenced, flat fielded, extracted, and wavelength calibrated using the SpeXTool IDL routines (Cushing et al. 2004). Telluric correction and absolute flux calibration were performed using a spectrum of a nearby A0V star obtained at similar air mass and time to PTF10nvg and reduced in similar fashion; they were applied using the XTELLCOR IDL package (Vacca et al. 2003). 
Table 4

Pre-outburst Photometry of PTF10nvg

\begin{tabular}{|c|c|c|c|c|c|}
\hline $\begin{array}{l}\text { Wavelength } \\
(\mu \mathrm{m})\end{array}$ & $\begin{array}{c}\text { Flux } \\
\text { Density (Jy) }\end{array}$ & $\begin{array}{c}\text { Flux } \\
\text { Error }(1 \sigma) \\
\end{array}$ & Observatory & Epoch & Reference \\
\hline 12 & 3.39 & 0.33 & IRAS & 1983 & Odenwald (1989) \\
\hline 25 & 6.59 & 0.66 & IRAS & 1983 & Odenwald (1989) \\
\hline 60 & 27.89 & 1.0 & $I R A S$ & 1983 & Odenwald (1989) \\
\hline 100 & 57.35 & 1.0 & IRAS & 1983 & Odenwald (1989) \\
\hline 8.28 & 1.6 & 0.66 & MSX & 1996-1997 & Egan et al. (1999) \\
\hline 12.13 & 2.49 & 0.137 & MSX & 1996-1997 & Egan et al. (1999) \\
\hline 14.65 & 3.045 & 0.189 & MSX & 1996-1997 & Egan et al. (1999) \\
\hline 21.34 & 3.14 & 0.201 & MSX & 1996-1997 & Egan et al. (1999) \\
\hline 3.6 & 0.083820 & 0.0041974110 & Spitzer/IRAC & 2006-2007 & Guieu et al. (2009) \\
\hline 4.5 & 0.231500 & 0.0011582866 & Spitzer/IRAC & 2006-2007 & Guieu et al. (2009) \\
\hline 5.8 & 0.609100 & 0.0030475698 & Spitzer/IRAC & 2006-2007 & Guieu et al. (2009) \\
\hline 8.0 & 1.171000 & 0.0058589830 & Spitzer/IRAC & 2006-2007 & Guieu et al. (2009) \\
\hline 24.0 & 2.222000 & 0.088946621 & Spitzer/MIPS & 2006-2007 & L. M. Rebull et al. 2011 (in preparation) \\
\hline 70.0 & 6.732000 & $\ldots$ & Spitzer/MIPS & 2006-2007 & L. M. Rebull et al. 2011 (in preparation) \\
\hline 9.0 & 1.96 & 0.2 & AKARI & 2006-2007 & Ishihara et al. (2010) \\
\hline 15.0 & 3.875 & 0.457 & AKARI & 2006-2007 & Ishihara et al. (2010) \\
\hline
\end{tabular}

TripleSpec observations were obtained by K.R.C. on 2010 July 18 with the 0.7 slit, providing nearly contiguous coverage over $1.0-2.5 \mu \mathrm{m}$ at $R \approx 5000$. Spectra were obtained and reduced in the same manner as the SpeX observations, using a version of the SpeXTool package modified for use with APO/ TripleSpec data.

A NIRSPEC spectrum covering $0.95-1.12 \mu \mathrm{m}$ at $R \approx 25,000$ was obtained on 2010 July 16 by L.A.H. Standard data-reduction steps including wavelength calibration and image rectification, extraction of one-dimensional spectra, and removal of telluric emission and absorption features were carried out by W.J.F. within the REDSPEC IDL package developed by S. S. Kim, L. Prato, and I. McLean.

\section{DISCOVERY AND CHARACTERIZATION}

PTF10nvg was first identified as an optical transient by the PTF automatic discovery and classification codes on 2010 July 8. It was selected for spectroscopic follow-up observation as a bright event observable with Keck during morning twilight of July 8, and subsequently visually identified as a likely outbursting young star. Analysis of the PTF data and the followup photometry and spectroscopy is presented below.

\subsection{Photometric Analysis}

\subsubsection{Outburst Characterization from the Optical/PTF Light Curve}

Although PTF10nvg is not known historically as an optical source, it was detected by PTF at $m_{R} \approx 18$ mag in mid-2009 but then faded to $\sim 19.25$ mag on a timescale of roughly 2 months. It brightened again by more than 4 mag in less than 200 days, while the field was too close to the Sun for observations. PTF10nvg reached $m_{R}=15$ early in 2010 , faded to $16.3 \mathrm{mag}$ within 40 days of the first peak, and then brightened again to $13.5 \mathrm{mag}$ before fading to $\sim 15.5 \mathrm{mag}$ in early 2010 November. The two brightness peaks, as well as the intermediate lull, are all $\sim 50$ days in width.

\subsubsection{Pre- and Post-outburst SED}

Pre-outburst photometry that we have collated from the literature is presented in Table 4, and the spectral energy distributions (SEDs) assembled from this pre-outburst photometry and our own post-outburst detection data are displayed in Figure 5.

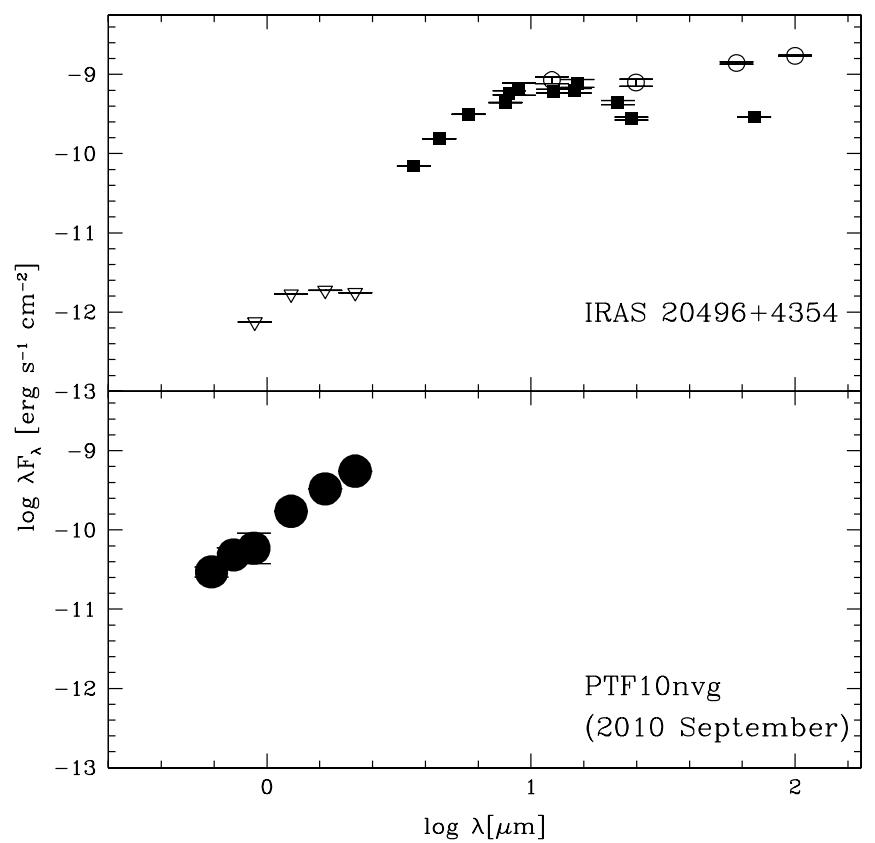

Figure 5. Pre-outburst (top panel) and post-outburst (bottom panel) SEDs of PTF10nvg. The pre-outburst data derive from KPNO $0.9 \mathrm{~m}$ optical and 2MASS NIR upper limits (triangles), Spitzer IRAC (3.6, 4.5, 5.8, 8.0 $\mu \mathrm{m}$; filled squares) and MIPS (24, $70 \mu \mathrm{m}$; filled squares), $\operatorname{MSX}(8.3,12.1,14.6,21.3 \mu \mathrm{m}$; filled squares), AKARI (9 and $18 \mu \mathrm{m}$; filled squares), and $I R A S(12,25,60,100 \mu \mathrm{m}$; open circles) photometric measurements (note that the IRAS beam was larger than that of the other instruments). The post-outburst data are those of 2010 July 9 as reported in Tables 1 and 3, when the object was in a relative lull between its two optical brightness maxima (see Figure 2).

From 3 to $10 \mu \mathrm{m}$, the SED is rising then becomes roughly flat from 10 to $100 \mu \mathrm{m}$. The integrated luminosity of the pre-outburst IR source between 3 and $100 \mu \mathrm{m}$ is $25 \mathrm{~L}_{\odot}$; extrapolating the SED longward of the IRAS $100 \mu \mathrm{m}$ measurement as a standard Rayleigh-Jeans function, and integrating to $\lambda=\infty$, increases the luminosity by $\sim 40 \%$. Other Class I sources, however, have SEDs that peak at or slightly longward of $100 \mu \mathrm{m}$ (e.g., Enoch et al. 2009); if PTF10nvg's SED possesses a similar peak, the total luminosity would be larger than this, which assumes a sharply declining power-law directly from $100 \mu \mathrm{m}$. The integrated luminosity of the outbursting source between 0.7 and 

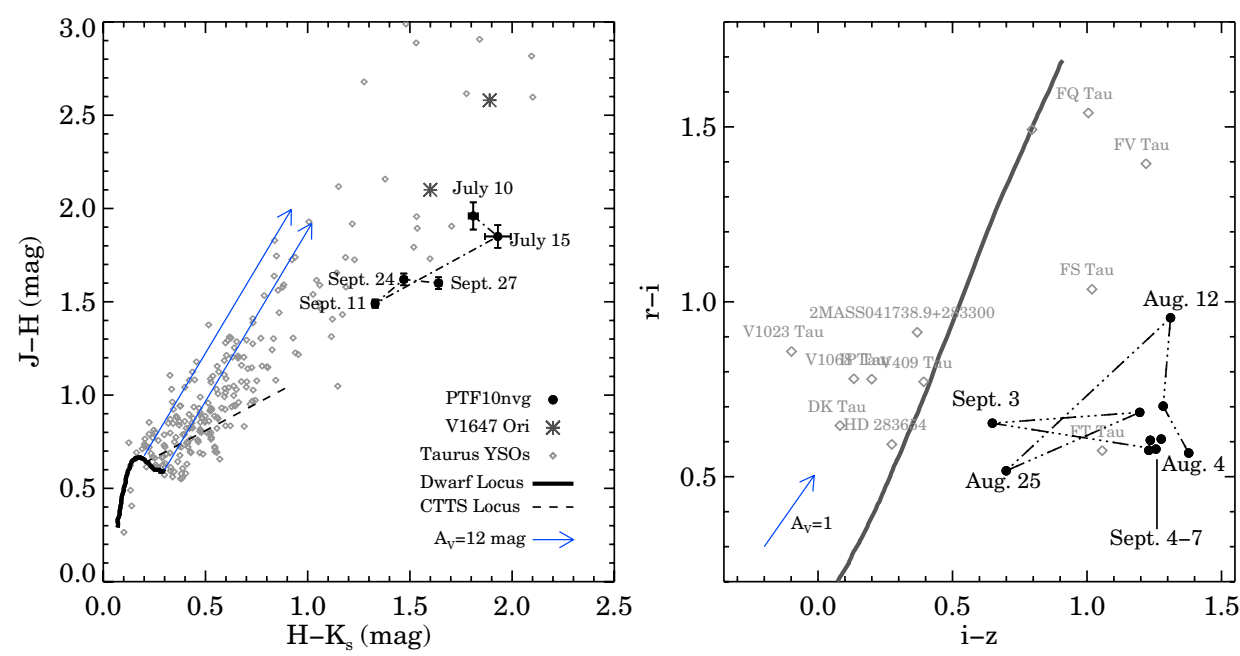

Figure 6. Location of PTF10nvg in $J H K_{s}$ (left) and riz (right) color-color diagrams. Solid lines in each panel indicate the locus of SDSS/2MASS colors for mainsequence field dwarfs (F to M spectral types; Covey et al. 2007); the dashed line in the top panel shows the Classical T Tauri locus derived by Meyer et al. (1997) and transformed into the 2MASS photometric system using the relationship derived by Carpenter (2001). Class I, II, and III objects in Taurus, as well as the outbursting object V1647 Ori, are shown for comparison; photometry for these comparison stars was catalogued by Finkbeiner et al. (2004), Reipurth \& Aspin (2004a), and Rebull et al. (2010).

(A color version of this figure is available in the online journal.)

$2.2 \mu \mathrm{m}$ is $2.5 L_{\odot}$, compared to $<0.016 L_{\odot}$ before the outburst. There is no post-outburst photometry longward of $2.2 \mu \mathrm{m}$.

The optical and NIR colors of PTF10nvg, shown in Figure 6, are extremely red during outburst: $r-i \approx 0.6 \mathrm{mag}, i-z \approx$ $1.1 \mathrm{mag}, J-H \approx 1.9 \mathrm{mag}$, and $H-K_{s} \approx 1.9 \mathrm{mag}$. These colors are within the distribution but toward the blue end of NIR colors exhibited by Class-I-type stars in Taurus, and significantly redder than can be explained by a range of disk accretion, inner hole, and inclination properties along the Class II "Classical T Tauri locus" (Meyer et al. 1997). In 2010 July, PTF10nvg appeared redder in $H-K_{s}$ and bluer in $J-H$ than most known "FU Ori-like" objects (e.g., see Figure 4 by Greene et al. 2008). As PTF10nvg began fading in 2010 September, however, its observed $J H K_{s}$ colors became somewhat bluer, comparable to the colors of V346 Nor, a previously known "FU Ori-like" object. The $J H K_{s}$ colors of PTF10nvg are also similar to those of V1647 in 2004 February-March, early in the 2004-2005 outburst.

High-energy radiation has been detected from other outbursting young stars (e.g., V1647; Kastner et al. 2006), but no X-ray detection can be confidently associated with PTF10nvg at this time. X-ray observations of PTF10nvg were obtained with the Swift X-Ray Telescope (XRT; Burrows et al. 2005) starting on 2010 August 29 for a total exposure time of $3.6 \mathrm{ks}$. We extract the $0.5-8.0 \mathrm{keV}$ counts from an extraction region of 64 pixels, $\sim 2.5$ arcmin, where we fit the point spread function model (see Butler \& Kocevski 2007) at the centroid of the optical position of PTF10nvg. We detect no X-ray emission from PTF10nvg. Assuming a $\Gamma=2$ power-law spectrum and a column density of $N_{\mathrm{H}}=10^{22} \mathrm{~cm}^{-2}$ we find a $3 \sigma$ upper limit of the flux: $F_{X}<1.2 \times 10^{-13} \mathrm{erg} \mathrm{cm}^{-2} \mathrm{~s}^{-1}$. Consistent with both the sensitivity of the ROSAT All Sky Survey (Voges et al. 1999) and the Swift upper limit, the closest ROSAT source is approximately 0.9 SW of PTF10nvg's optical position, suggesting that it is unlikely to be a counterpart to the star.

\subsection{Spectroscopic Analysis}

PTF10nvg exhibits a rich set of emission-lines, including hydrogen and both low- and high-excitation metallic atomic lines. Atomic line-strength differences between the multiple optical and IR observations are presented quantitatively in Tables 5 and 6. Equivalent widths (EWs) and line fluxes reported in these tables are calculated by integrating across the line after subtracting off the local continuum, which is estimated by interpolating between two line free regions well separated from the feature in question (typically $v>1000 \mathrm{~km} \mathrm{~s}^{-1}$ away). Line fluxes are simply calculated as the direct integral of the line in the flux calibrated spectra; EWs are calculated by dividing the integrated flux by the mean value of the local continuum.

In addition to the many atomic features in emission in PTF10nvg's spectrum, there is also strong molecular emission from $\mathrm{TiO}$ and $\mathrm{VO}$ at red optical wavelengths (see PTF10nvg's discovery spectrum, shown in Figure 7), and from VO, $\mathrm{H}_{2} \mathrm{O}$, and $\mathrm{CO}$ in the IR regime (see PTF10nvg's SpeX and TripleSpec spectra, shown in Figure 8). To the best of our knowledge, this is the first time such prominent molecular emission has been detected from a young star at optical wavelengths.

The four optical spectra from Keck, Lick, and Palomar are compared over the [O I] to Ca II triplet region in Figure 9. Note that the spectra were obtained after the first brightness peak in the 2010 light curve, near the beginning and the end of the $m_{R} \approx 16.5$ mag nadir (Keck and Lick) and then when the object was brightening again and had reached $m_{R} \approx 14.8$ mag for the second time during our monitoring. There is some evidence for a change in the overall continuum slope during this month; as the object brightened by several magnitudes it also became redder in a manner similar to interstellar extinction by $\sim 1$ mag in the blue and $\sim 1.5 \mathrm{mag}$ in the red. The emission feature strengths also varied, as discussed below. The broad $\mathrm{TiO}$ band emission, also discussed below, appeared strongest in the July 19 (Lick) spectrum just before the object began its second brightening.

We analyze spectroscopic features of particular interest in more detail below. To analyze the kinematic structure of the observed line profiles, we assume a rest velocity for PTF10nvg of $V_{\mathrm{LSR}} \sim 4 \mathrm{~km} \mathrm{~s}^{-1}$, consistent with ${ }^{13} \mathrm{CO}$ observations of the surrounding molecular gas (Dobashi et al. 1994).

\subsubsection{H I Lines}

PTF10nvg has strong $\mathrm{H}$ I emission lines including $\mathrm{H} \beta, \mathrm{H} \alpha$, the Paschen series (detectable up to 21), and the Brackett series 
Table 5

Optical Line Strengths

\begin{tabular}{|c|c|c|c|c|c|c|c|}
\hline Line & $\begin{array}{l}\text { Wavelength } \\
(\AA)\end{array}$ & $\begin{array}{c}\text { LRIS } \\
\text { EqW }(\AA) \\
\end{array}$ & $\begin{array}{c}\text { LRIS Flux } \\
\left(10^{-15} \mathrm{erg} \mathrm{cm}^{-2} \mathrm{~s}^{-1}\right) \\
\end{array}$ & $\begin{array}{l}\text { Kast (July) } \\
\text { EqW }(\AA)\end{array}$ & $\begin{array}{c}\text { Kast (July) Flux } \\
\left(10^{-15} \mathrm{erg} \mathrm{cm}^{-2} \mathrm{~s}^{-1}\right) \\
\end{array}$ & $\begin{array}{c}\text { DoubleSpec } \\
\text { EqW }(\AA)\end{array}$ & $\begin{array}{c}\text { DoubleSpec } \\
\text { Flux }\left(10^{-15} \mathrm{erg} \mathrm{cm}^{-2} \mathrm{~s}^{-1}\right) \\
\end{array}$ \\
\hline $\mathrm{H} \eta$ & 3835.38 & 3.68 & 0.087 & 4.15 & 0.21 & $\ldots$ & $\ldots$ \\
\hline $\mathrm{H} \zeta$ & 3889.05 & 2.57 & 0.14 & 6.57 & 0.30 & $\ldots$ & $\ldots$ \\
\hline Ca II K (abs) & 3933.66 & 1.41 & 0.056 & 4.01 & 0.25 & $\ldots$ & $\ldots$ \\
\hline Ca II K (em) & 3933.66 & -5.65 & 0.24 & -10.57 & 0.449 & $\ldots$ & $\ldots$ \\
\hline $\mathrm{H} \epsilon+$ Ca II $\mathrm{H}$ & 3970.07 & 4.93 & 0.21 & 7.04 & 0.366 & $\ldots$ & $\ldots$ \\
\hline $\mathrm{H} \delta$ & 4101.74 & 2.17 & 0.12 & 5.34 & 0.306 & $\ldots$ & $\ldots$ \\
\hline $\mathrm{H} \gamma$ & 4340.47 & 4.00 & 0.33 & 7.13 & 0.57 & $\ldots$ & $\ldots$ \\
\hline $\mathrm{H} \beta$ (abs) & 4861.33 & 2.88 & 0.46 & 3.92 & 0.55 & $\ldots$ & $\ldots$ \\
\hline $\mathrm{H} \beta$ (em) & 4861.33 & -1.7 & 0.27 & -0.3 & 0.04 & $\ldots$ & $\ldots$ \\
\hline $\mathrm{NaD}$ & $5889.95+5895.92$ & 9.637 & 4.35 & 7.82 & 2.85 & $\ldots$ & $\ldots$ \\
\hline Fe I & 6191.56 & -0.61 & 0.368 & -1.82 & 0.89 & $\ldots$ & $\ldots$ \\
\hline [O I] & 6300.30 & -9.14 & 5.48 & -7.91 & 3.99 & $\ldots$ & $\ldots$ \\
\hline [O I] & 6363.78 & -3.8 & 2.245 & -2.95 & 1.41 & -1.5 & 2.22 \\
\hline Fe II & 6432.68 & -2.11 & 1.33 & -3.38 & 1.59 & -1.68 & 2.73 \\
\hline $\mathrm{Fe}_{\mathrm{I}}$ & 6495.74 & -1.158 & 0.80 & -2.27 & 1.17 & -1.78 & 2.97 \\
\hline $\mathrm{Fe}$ II & 6516.08 & -2.13 & 1.5 & -2.84 & 1.49 & -2.92 & 4.89 \\
\hline $\mathrm{H} \alpha$ & 6562.85 & -39.9 & 31.5 & -56.5 & 31.33 & -24.7 & 48.6 \\
\hline$[\mathrm{N}$ II $]$ & 6592 & -0.86 & 0.70 & -0.18 & 0.13 & -1.15 & 2.23 \\
\hline [S II] & 6716.44 & -0.34 & 0.29 & 1.26 & 0.89 & $\ldots$ & $\ldots$ \\
\hline [S II] & 6730.82 & -0.80 & 0.67 & 0.82 & 0.58 & $\ldots$ & $\ldots$ \\
\hline $\mathrm{Ca} \mathrm{I}$ & 7148.15 & -1.83 & 2.04 & -1.76 & 1.64 & $\ldots$ & $\ldots$ \\
\hline [Fe II] & 7172.00 & -0.59 & 0.66 & 0.58 & 0.56 & $\ldots$ & $\ldots$ \\
\hline [Ca II] & 7291.47 & -1.08 & 1.23 & -1.63 & 1.46 & -1.14 & 3.47 \\
\hline [Ca II] & 7323.89 & -1.02 & 1.13 & -1.12 & 0.97 & -1.19 & 3.6 \\
\hline $\mathrm{K}_{\mathrm{I}}$ & 7664.91 & 3.02 & 4.22 & 2.24 & 2.66 & 3.16 & 11.1 \\
\hline $\mathrm{K}_{\mathrm{I}}$ & 7698.96 & 2.32 & 3.32 & 2.86 & 3.80 & 1.54 & 5.74 \\
\hline $\mathrm{O}_{\mathrm{I}}$ & 7773 & 2.26 & 3.42 & 2.43 & 3.35 & 1.77 & 7.32 \\
\hline $\mathrm{O}_{\mathrm{I}}$ & 8446.36 & -1.52 & 2.86 & -2.47 & 3.98 & -2.16 & 9.81 \\
\hline Ca II & 8498.02 & -16.8 & 32.8 & -23.46 & 35.57 & -19.0 & 85.6 \\
\hline Ca II & 8542.09 & -19.1 & 35.9 & -24.24 & 37.63 & -16.2 & 69.3 \\
\hline [Fe II] & 8616.952 & -1.14 & 2.1 & -0.73 & 1.11 & $\ldots$ & $\ldots$ \\
\hline Ca II & 8662.14 & -15.4 & 29.0 & -24.88 & 36.7 & -17.55 & 60.8 \\
\hline Н I (Pa 11) & 8863.4 & -1.47 & 2.64 & -3.14 & 4.14 & $\ldots$ & $\ldots$ \\
\hline $\mathrm{HI}(\mathrm{Pa} 10)$ & 9015.6 & -1.58 & 2.98 & -2.15 & 2.80 & $\ldots$ & $\ldots$ \\
\hline Н I (Pa 9) & 9229.7 & -1.61 & 3.46 & -2.73 & 4.26 & $\ldots$ & $\ldots$ \\
\hline Н I (Pa 8) & 9545.97 & -1.44 & 3.36 & -3.59 & 5.19 & $\ldots$ & $\ldots$ \\
\hline$[\mathrm{Fe} \mathrm{I}]$ & 9998.33 & -2.28 & 5.21 & $\ldots$ & $\ldots$ & $\ldots$ & $\ldots$ \\
\hline H I (Pa 7) & 10049.4 & -1.82 & 4.89 & -6.35 & 9.3 & $\ldots$ & $\ldots$ \\
\hline
\end{tabular}

including $\mathrm{Br}-\alpha$ at $4.05 \mu \mathrm{m}$. No Balmer jump is apparent, but the pileup of the higher-order Brackett lines is visible in the $H$ band of the SpeX observations. The high-dispersion NIRSPEC spectrum reveals profile widths in the $\mathrm{Pa}-\gamma$ and $\mathrm{Pa}-\delta \mathrm{HI}$ lines of $\sim 125 \mathrm{~km} \mathrm{~s}^{-1}$ FWHM. The lines are slightly asymmetric with respect to their peak emission at roughly zero velocity, having somewhat more integrated flux on the blue side of line center than on the red side. The $\mathrm{H} \alpha$ line EWs are -26 to $-46 \AA$ among the four optical spectra, moderate but not extreme relative to Taurus Class I or Class II objects (White \& Hillenbrand 2004).

We have derived an estimate of the extinction towards PTF10nvg from the strength of its $\mathrm{Pa} \delta$ and $\mathrm{Br} \gamma$ emission lines. These lines, representing the $7-3$ and $7-4$ transitions respectively, share the same upper level. Neglecting collisional effects (a questionable assumption that we will return to below), the relative frequency of radiative de-excitations from the same upper level should be determined entirely by atomic physics. That is, the intrinsic flux ratio of these lines can be calculated in the collisionless limit as

$$
\frac{F_{B r \gamma}}{F_{P a \delta}}=\frac{A_{B r \gamma} \lambda_{P a \delta}}{A_{P a \delta} \lambda_{B r \gamma}},
$$

where $\mathrm{F}, \mathrm{A}$, and $\lambda$ give the flux, Einstein coefficient, and wavelength of each line. Using line constants from the NIST atomic database, we calculate an intrinsic $\mathrm{Br} \gamma$-to- $\mathrm{Pa} \delta$ line ratio of 0.42 for a collisionless emission region. Densities in magnetospheric accretion columns, however, are sufficiently high that collisions are likely to be an important factor for the emergent fluxes of $\mathrm{H}$ I emission lines. Detailed radiative transfer models of relatively cool $\left(T_{\max }=6000 \mathrm{~K}\right)$ magnetospheric accretion columns nonetheless return a $\mathrm{Br} \gamma$-to- $\mathrm{Pa} \delta$ line ratio very close to this naive calculation. Models of significantly warmer $\left(T_{\max }=9000 \mathrm{~K}\right)$ accretion columns return a larger line ratio of 2.21 (R. Kurosawa 2010, private communication). The line strength ratio in the SpeX spectrum of PTF10nvg is $\sim 8.2$, demonstrating the presence of significant extinction relative to either accretion model. We therefore estimate the extinction to the $\mathrm{H}$ I line emission region (which may or may not be coincident with the stellar photosphere) by assuming a standard $R_{V}=$ 3.1 extinction law, and de-reddening the observed line fluxes to match each of these predicted intrinsic line ratios. These estimates place crude bounds on the extinction to PTF10nvg's emission line region of $6<A_{V}<12.4$. 


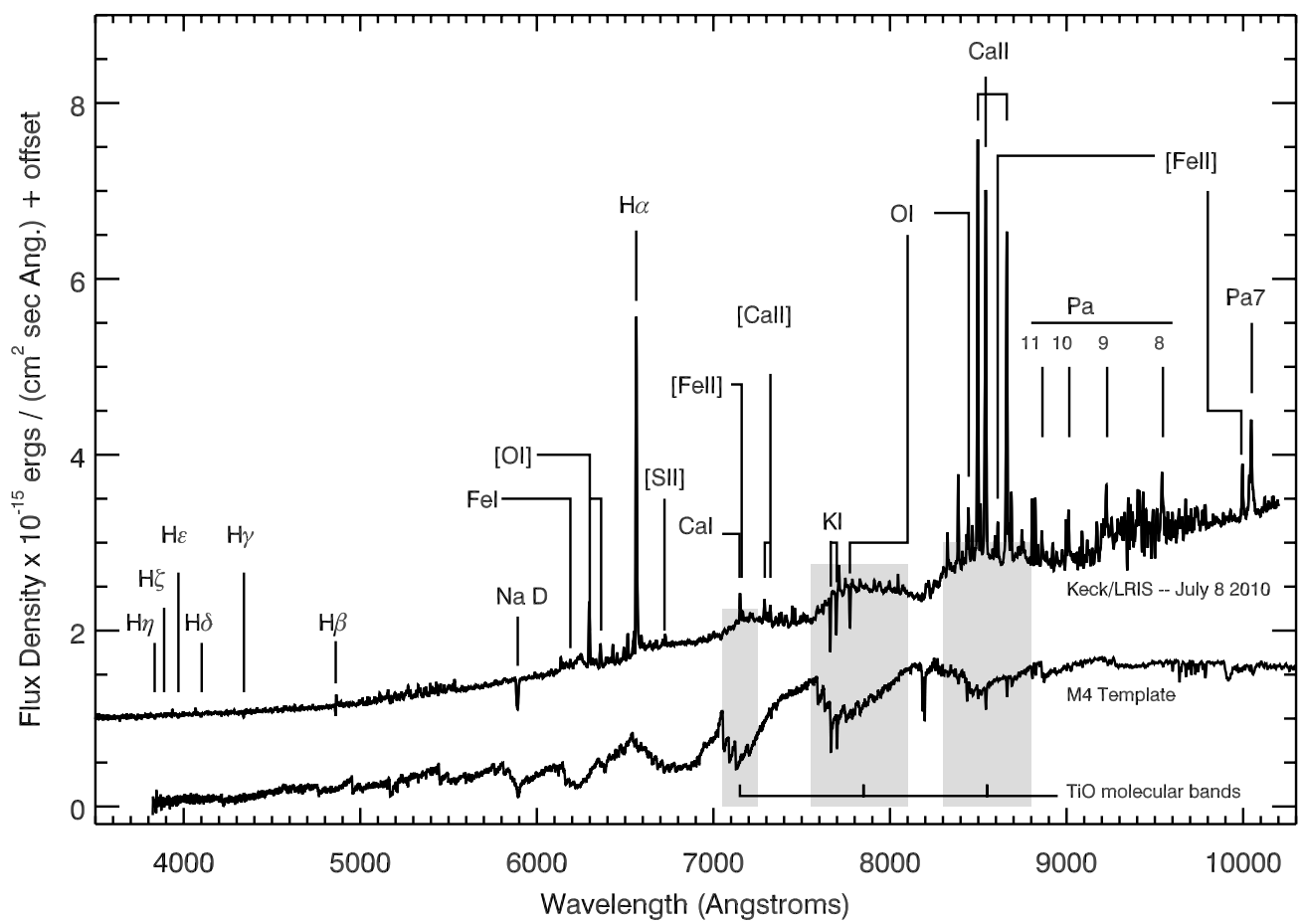

Figure 7. Optical spectrum of PTF10nvg obtained with Keck/LRIS on 2010 July 8 . Strong emission lines are identified, with line strengths presented quantitatively in Table 5. An M4 field-dwarf spectral template (Bochanski et al. 2007) is shown to illustrate the correspondence between the TiO emission bands in PTF10nvg and typical $\mathrm{TiO}$ absorption bands in cool M-type photospheres.

Table 6

Near-infrared Line Strengths

\begin{tabular}{|c|c|c|c|c|c|}
\hline Line & $\begin{array}{l}\text { Wavelength } \\
(\mu \mathrm{m})\end{array}$ & $\begin{array}{c}\text { SpeX } \\
\text { EqW }(\AA)\end{array}$ & $\begin{array}{c}\text { SpeX Flux } \\
\left(10^{-15} \mathrm{erg} \mathrm{cm}^{-2} \mathrm{~s}^{-1}\right) \\
\end{array}$ & $\begin{array}{l}\text { TripleSpec } \\
\text { EqW }(\AA)\end{array}$ & $\begin{array}{c}\text { TripleSpec Flux } \\
\left(10^{-15} \mathrm{erg} \mathrm{cm}^{-2} \mathrm{~s}^{-1}\right) \\
\end{array}$ \\
\hline Ca II & 0.8662 & -12.91 & -26.4 & $\ldots$ & $\ldots$ \\
\hline Ca II & 0.8542 & -13.49 & -28.5 & $\ldots$ & $\ldots$ \\
\hline Ca II & 0.8499 & -13.97 & -30.73 & $\ldots$ & $\ldots$ \\
\hline Н I $7-3$ & 1.0049 & -0.698 & -1.706 & -4.04 & -7.10 \\
\hline H I 6-3 & 1.0938 & -3.14 & -8.625 & -16.01 & -19.4 \\
\hline O I & 1.1287 & -4.211 & -12.397 & -13.01 & 10.06 \\
\hline Fe I & 1.1595 & -0.733 & -2.293 & -1.15 & -2.29 \\
\hline $\mathrm{Fe}_{\mathrm{I}}$ & 1.161 & -1.294 & -4.152 & -3.30 & -6.83 \\
\hline $\mathrm{K}_{\mathrm{I}}$ & 1.169 & -1.449 & -4.539 & -3.13832 & -6.80 \\
\hline $\mathrm{Fe} I$ & 1.179 & -0.807 & -2.665 & -1.45 & -3.55 \\
\hline $\mathrm{Mg}_{\mathrm{I}}$ & 1.1833 & -2.092 & -6.881 & -2.64 & -6.16 \\
\hline $\mathrm{Fe}_{\mathrm{I}}$ & 1.1885 & -3.018 & -10.106 & -5.26 & -12.47 \\
\hline $\mathrm{Fe}_{\mathrm{I}}$ & 1.1974 & -1.847 & -6.444 & -3.48 & -8.89 \\
\hline Н г $5-3$ & 1.2818 & -7.766 & -31.026 & -16.5 & -50.4 \\
\hline $\operatorname{Mg}_{I}$ & 1.504 & -3.777 & -23.54 & -5.39 & -29.7 \\
\hline $\mathrm{Si}$ I & 1.589 & -2.372 & -16.094 & -2.47 & -15.3 \\
\hline $\operatorname{Mg}_{I}$ & 1.711 & -0.587 & -4.73 & -0.73 & -5.50 \\
\hline $\mathrm{H}_{2}(1-0 \mathrm{~S}(1))$ & 2.121 & -0.848 & -10.12 & -1.35 & -16.9 \\
\hline Н I $7-4(\mathrm{Br} \gamma)$ & 2.1657 & -1.186 & -14.40 & -2.24 & -28.58 \\
\hline $\mathrm{Na} I$ & 2.2075 & -0.768 & -9.53 & -0.957 & -12.49 \\
\hline $\mathrm{H}_{2}(2-1 \mathrm{~S}(1))$ & 2.247 & -0.199 & -2.412 & -0.41 & -5.52 \\
\hline $\mathrm{Ca} \mathrm{I}$ & 2.282 & -0.503 & -6.5 & -1.07 & -14.9 \\
\hline $\mathrm{CO}$ & 2.2923 & -18.8 & -249.9 & -29.3 & -423.9 \\
\hline
\end{tabular}

Methods have also been developed to estimate a young star's mass accretion rate from the strength of its NIR HI emission lines (e.g., Muzerolle et al. 1998). These relationships, which link observed $\mathrm{H}$ I line luminosities with mass accretion rates inferred from UV continuum excesses, have only been calibrated for observations of T Tauri stars; applying these relationships to more heavily embedded protostars requires the assumption that the dynamics of the underlying accretion process are similar across these evolutionary phases, and that the line luminosity can be measured accurately even when the protostar's photosphere is not detectable. These assumptions are even more problematic for an extreme accretor such as PTF10nvg, but an accretion rate estimate via $\mathrm{HI}_{\mathrm{I}}$ line fluxes can still provide a useful lower limit on the object's accretion rate: changes in the accretion dynamics (i.e., boundary-layer accretion instead of magnetospheric accretion) or the presence of significant veiling 

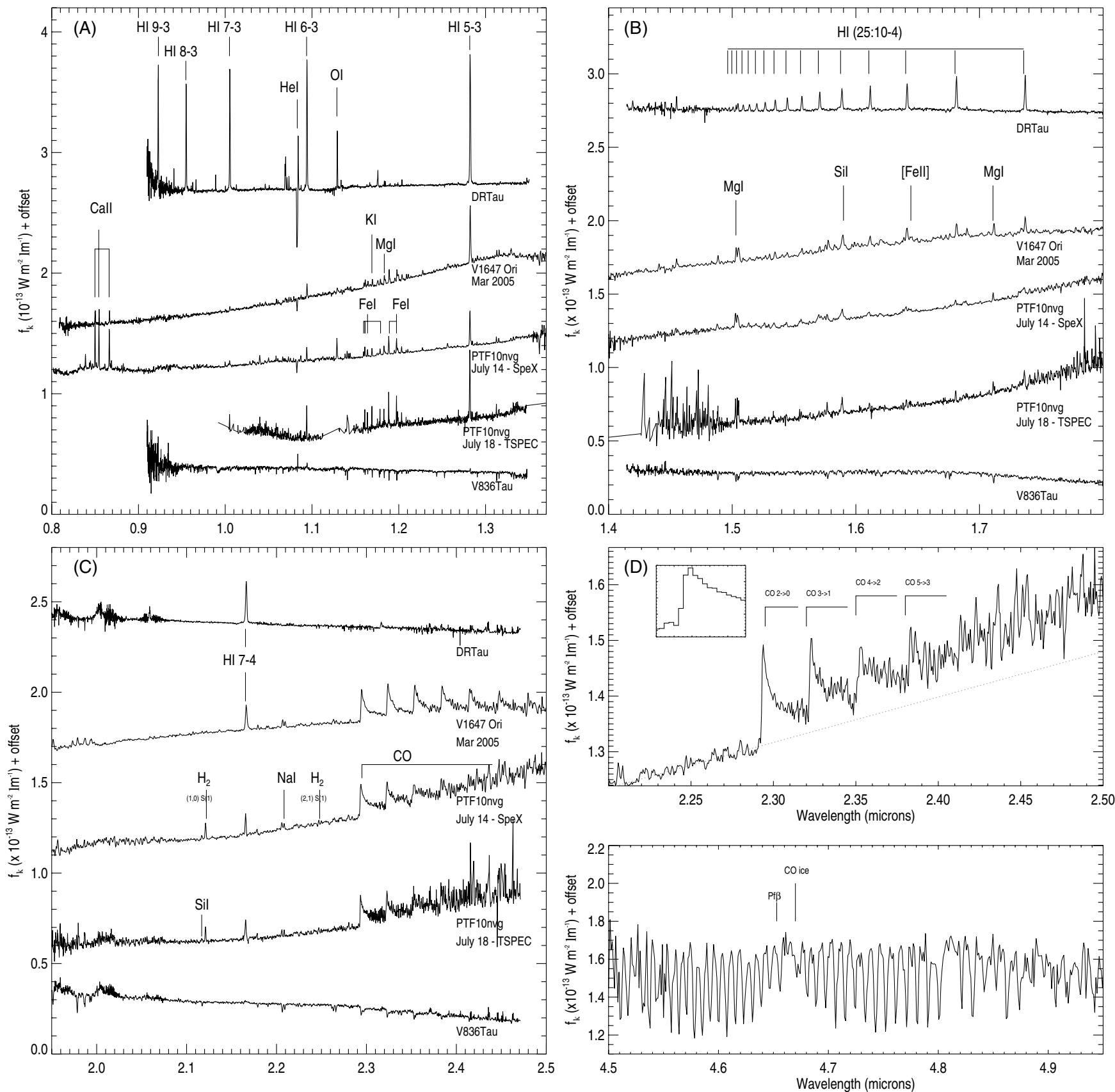

Figure 8. $Y J$-, $H$-, $K$-, and $M$-band spectra of PTF10nvg in panels (A), (B), (C), and (D), respectively. Panels (A)-(C) also include spectra of DR Tau (heavily accreting Class II object), V836 Tau (typical Class III object), and V1647 Ori (McNeil's Nebula outburst) for comparison. Strong emission lines are labeled where possible; PTF10nvg line strengths are also presented quantitatively in Table 6. Notably, the EWs of the emission lines in PTF10nvg are 50\%-100\% larger in the APO TripleSpec observation than in the SpeX observation even though the line fluxes are similar. The difference in EWs therefore likely reflects differences in the continuum flux level between the two epochs.

flux will both likely result in an underestimate the object's full line flux, and/or an increase in the conversion factor between line luminosities and accretion rates.

We have generated crude mass accretion rate estimates for PTF10nvg from the strengths of its $\mathrm{Br}-\gamma$ and $\mathrm{Pa}-\beta$ emission lines. We have dereddened the $\mathrm{Pa}-\beta$ and $\mathrm{Br}-\gamma$ line fluxes listed in Table 6 assuming an $A_{V} \approx 9 \mathrm{mag}$ (intermediate to the bounds calculated above), and converted those dereddened fluxes into line luminosities assuming a distance to PTF10nvg of $\sim 600 \mathrm{pc}$. Using Equations (1) and (2) from Muzerolle et al. (1998) to estimate $\log L_{\text {acc }} / L_{\odot}$ from each line, we infer $\log L_{\text {acc }} / L_{\odot}$ $=0.62$ and 0.335 from the $\mathrm{Pa}-\beta$ and $\mathrm{Br}-\gamma$ lines, respectively.
Assuming canonical Classical T Tauri Star parameters $(M=$ $0.5 M_{\odot}$ and $R=3 R_{\odot}$ ), these accretion luminosities imply mass accretion rates of $\dot{M} \approx 2.5 \times 10^{-7} M_{\odot} \mathrm{yr}^{-1}$.

\subsubsection{Ca II}

The Ca II triplet lines at 8498,8542 , and $8662 \AA$ are also generally associated with accretion activity in young stars. PTF10nvg exhibits EWs for these lines of -15 to $-25 \AA$, varying among the observations, but in the realm of the rapidly accreting young Class II stars in Taurus and comparable to the emission levels measured by Connelley \& Greene (2010) in a large survey of Class I objects. The line ratios seen 


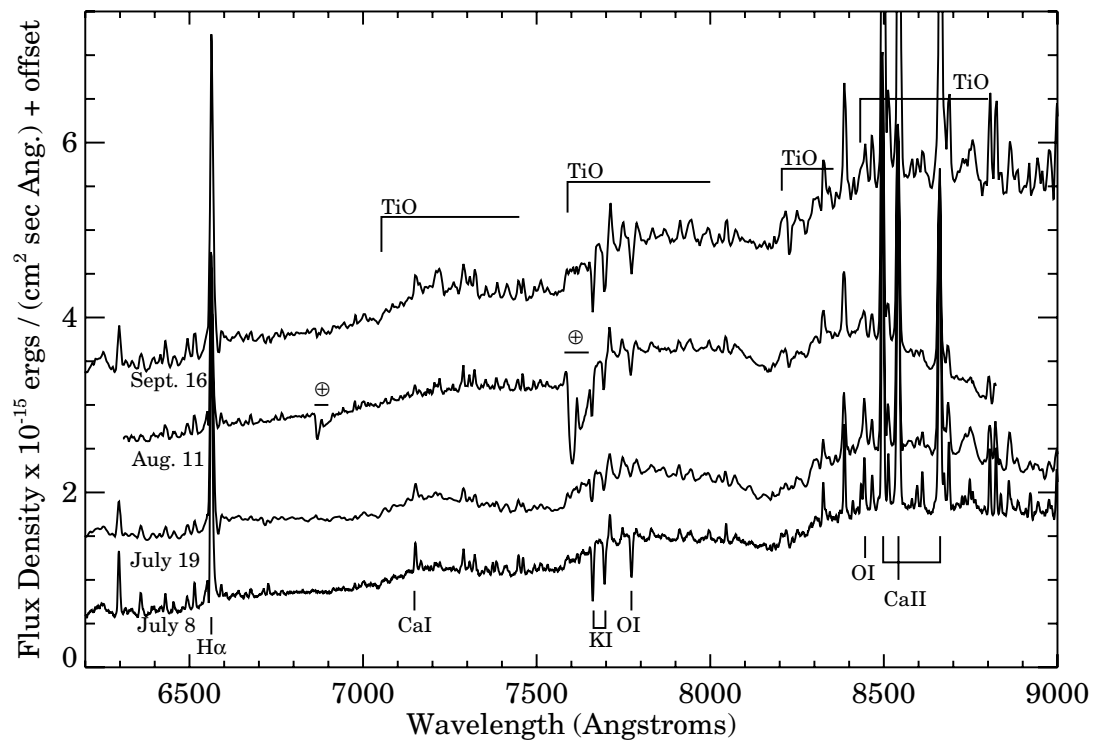

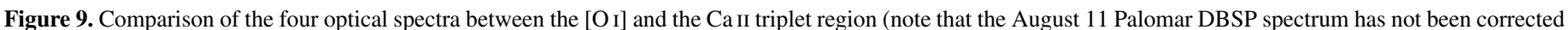
for telluric absorption. The apparent "continuum" differences are due to variations in the strength of the TiO emission over the four epochs.

from PTF10nvg varied, being somewhat typical of Class II sources $\left(F_{\mathrm{Ca} \text { II8498 }} / F_{\mathrm{CaII8542}}=0.91, F_{\mathrm{Ca} \mathrm{II8662}} / F_{\mathrm{CaII8542}}=0.81\right)$ in the LRIS data taken on 2010 July 8, but roughly equal $\left(F_{\text {Ca II8498 }} / F_{\text {Ca II8542 }}=1.08, F_{\text {Ca II8662 }} / F_{\text {Ca II8542 }}=0.93\right)$ in the SpeX data from July 14 . The latter case is again similar to heavily accreting $\mathrm{T}$ Tauri stars at the extreme of the Class II range illustrated by Hamann \& Persson (1992) and close to the ratio predicted by their highly optically thick $(\tau=1000)$ slab models. It is also similar the outbursting behavior of V1647 Ori (Walter et al. 2004).

Like the Paschen lines, the Ca II triplet lines appear slightly asymmetric with respect to their line centers, having somewhat more flux on the blueshifted sides of the lines than the red.

The Ca II $\mathrm{K}$ line is also in emission, though the $\mathrm{H}$ line is affected by blueshifted Balmer line absorption.

\subsubsection{Other Atomic Lines}

The only absorption features we detect in these optical spectra are blueshifted lines of $\mathrm{H} \mathrm{I}, \mathrm{Na}$ I D, and $\mathrm{K}$ I, along with the O I triplet at $7773 \AA$, which appears centered at the systemic velocity. The IR spectra, similarly, have only a single blueshifted line in absorption (the He I $\lambda 10830$ feature seen in Figure 8 and discussed in Section 3.2.4), so no spectral type can be derived for the outbursting object. Instead of a stellar photosphere, the spectrum is dominated by the circumstellar signatures of accretion and outflow processes.

In addition to the $\mathrm{H}$ I and $\mathrm{Ca}$ II lines discussed in Sections 3.2.1 and 3.2.2, the optical and NIR spectra of PTF10nvg show many emission lines characteristic of young accreting stars. The optical spectra show several permitted and forbidden species, including $\mathrm{O}$ I, Fe I, Fe II, Ca I, Ca II, [Fe II], [Ca II], and [S II]. The high-dispersion Keck NIRSPEC $Y$-band spectrum shows low-excitation $(<1-2 \mathrm{eV})$ Ti I lines as well as higher excitation (6-8 eV) $\mathrm{Si}$ I and $\mathrm{C}$ I lines in abundance, identified from the line list presented by Sharon et al. (2010). A $Y$-band survey including many Class II and some Class I stars in Taurus, as well as other young stars (Edwards et al. 2006; Fischer et al. 2008), suggests that some extreme emission line objects display the higher excitation lines. The $\mathrm{Ti}$ I is quite rare, however, and is found in our experience only in the spectra of V1331
Cyg, ZCMa, and SVS 13-all embedded sources driving strong outflows. The lower dispersion SpeX spectrum shows emission lines from many neutral species including $\mathrm{Al}$ I, Ca I, Fe I, K I, $\mathrm{Mg}$ I, Na I, O I, Si I, and Ti I over the 1-2.5 $\mu \mathrm{m}$ region; many of these features are also visible in the NIR outburst spectrum of V1647 (shown for comparison in Figure 8).

\subsubsection{Wind Indicators: Blueshifted H I, Na D, KI, and He I $\lambda 10830$ Absorption and [ $\left.\mathrm{O}_{\mathrm{I}}\right]$ Emission}

There is significant evidence for a substantial wind associated with the outburst of PTF10nvg. Our optical spectra have limited resolution of $\sim 150-300 \mathrm{~km} \mathrm{~s}^{-1}$, but nevertheless the $\mathrm{H} \alpha$ and $\mathrm{H} \beta$ lines display clear $\mathrm{P}$ Cygni features with sub-continuum absorption out to $-400 \mathrm{~km} \mathrm{~s}^{-1}$ (see kinematic profiles shown in Figure 10). The upper Balmer series lines lack the redshifted emission and display only a broad, blueshifted absorption trough. In the NIR, where our NIRSPEC spectrum provides a higher resolution by a factor of $2-3$, none of the Paschen or Brackett lines has P Cygni structure. The Na I $\mathrm{D}$ lines, like the upper Balmer lines, are seen in blueshifted absorption out to about $-400 \mathrm{~km} \mathrm{~s}^{-1}$ but are blended at our resolution.

The K I doublet at 7664 and $7698 \AA$ is seen in absorption in PTF10nvg's spectrum, blueshifted by $\sim 175 \mathrm{~km} \mathrm{~s}^{-1}$; this line is not typically seen in the spectra of Class I or Class II stars. Strong and blueshifted K I and Na I D absorption, as well as $\mathrm{P}$ Cygni Balmer profiles, are seen in the optical spectra of the FU Ori stars V1057 Cyg and V1515 Cyg, however, and in the outburst spectrum of V1647 Ori.

The high-dispersion NIRSPEC spectrum shows a somewhat complex He I $\lambda 10830$ profile (see Figure 11). Not unlike many heavily accreting T Tauri stars, such as DR Tau (Edwards et al. 2003, 2006), there is strong blueshifted absorption out to about $-300 \mathrm{~km} \mathrm{~s}^{-1}$ which reaches $60 \%$ of the continuum level. The absorption does not extend continuously inward to zero velocity, however; instead, the continuum level is reached by about $-100 \mathrm{~km} \mathrm{~s}^{-1}$ and there is a second narrow (FWHM $\approx$ $17 \mathrm{~km} \mathrm{~s}^{-1}$ ) absorption component centered at PTF10nvg's rest velocity. Similar low-velocity and narrow absorption components are seen in the weakly or nonaccreting T Tauri stars V836 


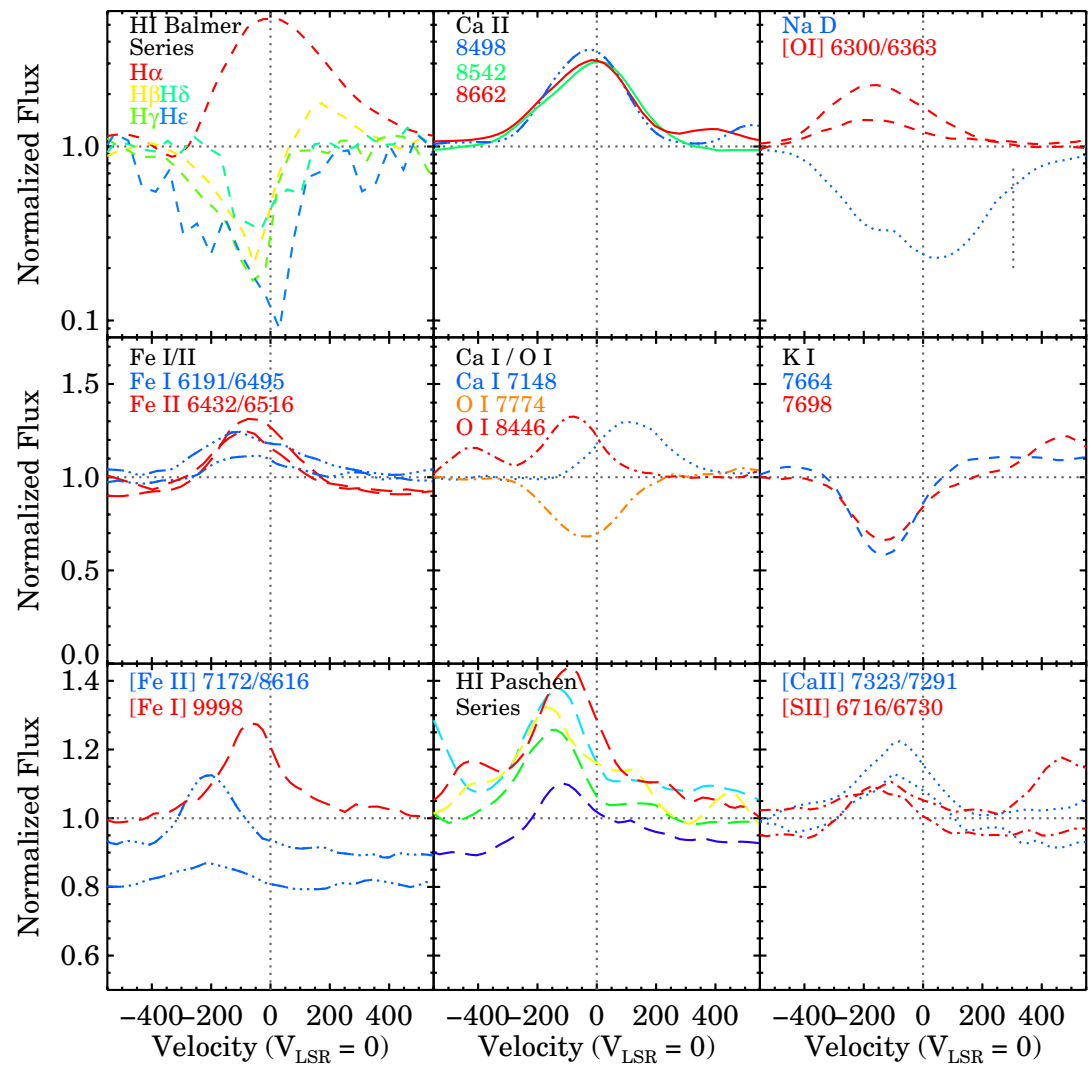

Figure 10. Selected spectral line profiles from the Keck LRIS spectrum, displayed with a velocity scale relative to the rest velocity of the Pelican Nebula $\left(V_{\mathrm{LSR}} \approx\right.$ $\left.0 \mathrm{~km} \mathrm{~s}^{-1}\right)$.

(A color version of this figure is available in the online journal.)

Tau and TWA 2. When considering both the broad absorption terminal velocity and the narrow absorption depth, the He I $\lambda 10830$ profile of PTF10nvg is quite similar to that of V1057 Cyg (unpublished data). The origin of a narrow absorption feature at roughly rest relative to the star is unknown; we note, however, the similar feature of V836 Tau was attributed to a disk wind by Kwan et al. (2007). Also of note is the possible presence of Si I emission at $10830 \AA$ that could be filling in some of the blueshifted He I absorption at intermediate velocities; this can be gauged by comparison to the Si I $\lambda 10869$ line also shown in Figure 11.

Other typical indicators of mass loss (Hartigan et al. 1995) are the $[\mathrm{O}$ I] $\lambda \lambda 6300,6364$ emission lines, which in PTF10nvg are symmetric and blueshifted by $\sim 175 \mathrm{~km} \mathrm{~s}^{-1}$, and the [S II] $\lambda \lambda 6717,6731$ lines, also blueshifted by $\sim 175 \mathrm{~km} \mathrm{~s}^{-1}$. The [N II] $\lambda \lambda 6548,6584$ lines are relatively weak and [O I] $\lambda 5577$ is not apparent in our low spectral resolution data. The forbidden-line EWs are comparable to those seen in the some of the strongest Class II stars in Taurus such as CW Tau, DD Tau, DG Tau, HN Tau, and UZ TauE (Hartigan et al. 1995), but weaker than those typical of Class I and other stars in Taurus driving strong outflows (White \& Hillenbrand 2004).

Hartigan et al. (1995) used detailed shock models to derive relations allowing a young star's mass loss rate to be estimated from the luminosities of its [O I] 6300 and [S II] 6731 forbidden lines. These relations are based upon assumptions about the typical density, velocity, and abundance of the shock region, and also require that observed line fluxes be converted to line luminosities, with corrections to account for reddening. Given the strong outflow signatures PTF10nvg possesses, it is possible, and perhaps likely, that the parameters commonly assumed for $\mathrm{T}$ Tauri star jets are not a good description of the physical properties of shocked regions in PTF10nvg's outflow. Nonetheless, we can convert the observed [OI] 6300 and [S II] 6716 line fluxes to luminosities by adopting the same distance and extinction estimates used above to calculate $\mathrm{HI}$ line luminosities, and thus infer crude estimates of PTF10nvg's mass loss rate from Hartigan, Edwards \& Ghandour's (1995) equations $\mathrm{A} 8$ and $\mathrm{A} 10$. These calculations imply estimated mass loss rates of $7 \times 10^{-7} M_{\odot} \mathrm{yr}^{-1}$ (as inferred from [S II] 6716) and $2 \times 10^{-6} M_{\odot} \mathrm{yr}^{-1}$ (as inferred from [O I] 6300). Given the sizable uncertainties in the adopted extinction correction, and even the appropriateness of the physical properties assumed for the shock regions, these mass loss rates may easily be incorrect by more than an order of magnitude. Nonetheless, they do emphasize the considerable mass loss associated with the PTF10nvg outburst; relative to the $\sim 10^{-7} M_{\odot} \mathrm{yr}^{-1}$ accretion rate inferred above from the $\mathrm{H}$ I emission lines, these estimates suggest an inflow/outflow ratio $\gtrsim 1$, one to two orders of magnitude larger than the $0.01-0.1$ ratios typically inferred for T Tauri stars.

NIR line ratios provide additional evidence that PTF10nvg is driving a strong outflow. Lorenzetti et al. (2009) and Antoniucci et al. (2008) have noted that EXor/jet driving sources typically demonstrate flux ratios between their $\mathrm{Br}-\gamma$ and $2.206 \mu \mathrm{m} \mathrm{Na}$ I emission lines of $\sim 2-5$. Class I sources that lack jet signatures, however, tend to have ratios that favor $\mathrm{Br}-\gamma$ much more strongly (e.g., 6-20; Nisini et al. 2005). The NIR spectra of PTF10nvg presented here possess a ratio of these lines of $\sim 2$, placing PTF10nvg firmly in the company of the jet driving sources, as is consistent with the morphology of the wind-sensitive lines analyzed above. 


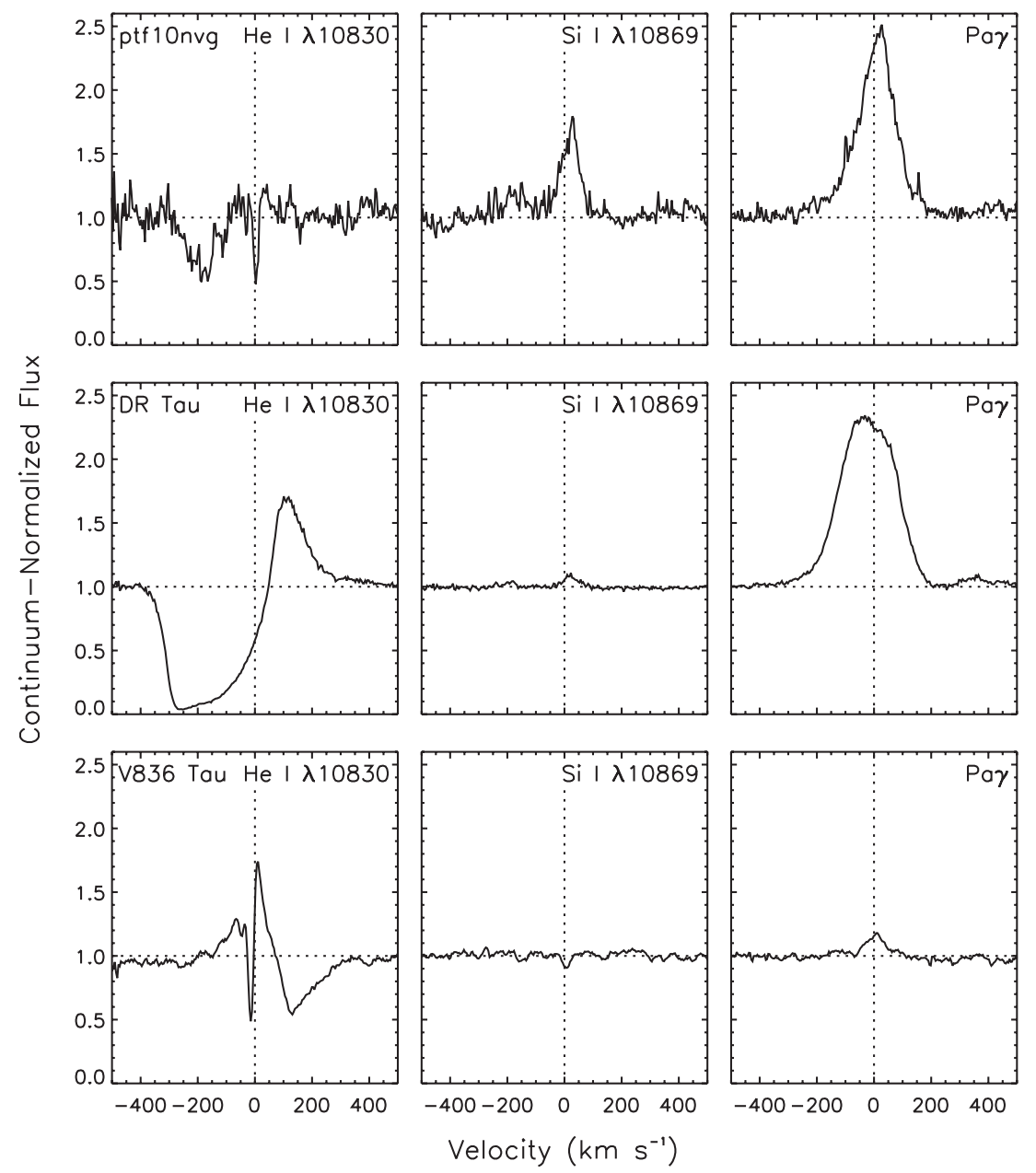

Figure 11. Keck NIRSPEC profiles of He I $\lambda 10830$, Si I $\lambda 10869$ in order to assess the possible contamination of Si I $\lambda 10830$ to the He I profile, and the H I Pa- $\gamma$ line of PTF10nvg. DR Tau and V836 Tau are shown for comparison: the two Taurus stars are benchmarks for their comparable terminal velocity in the broad blueshifted absorption (DR Tau) and the roughly systemic narrow absorption (V836 Tau) components to the He I profile.

\subsubsection{Outflow as Diagnosed Via $\mathrm{H}_{2}$ and [Fe II] Emission}

PTF10nvg shows clear emission in the $2.12 \mu \mathrm{m} \mathrm{H}_{2}(1-0) S(1)$ line, as well as a weaker feature which is likely the $2.24 \mu \mathrm{m}$ $S(2-1) S(1)$ line. Modeling has established that the ratio of these line strengths can diagnose between collisional and radiative excitation mechanisms: pioneering work by Gredel \& Dalgarno (1995) demonstrated that UV and X-ray irradiation will produce distinctive $\mathrm{H}_{2}(1-0) S(1) / S(2-1) S(1)$ line ratios ( 0.54 and 0.06 , respectively), and could also be distinguished from the ratio expected from a $T=2000 \mathrm{~K}$ shocked gas (0.13). More recently, Nomura et al. (2007) calculated synthetic $\mathrm{H}_{2}$ spectra as expected to emerge from protoplanetary disks with realistic gas-to-dust ratios and density/temperature profiles. The Nomura et al. (2007) models predict that $\mathrm{H}_{2}$ excitation by UV radiation is sensitive to the dust properties in the disk, producing $\mathrm{H}_{2}(1-0) S(1) / S(2-1) S(1)$ line ratios from $0.025-0.23$ for power-law distributions of dust grains with maximum sizes of $10 \mu \mathrm{m}$ to $10 \mathrm{~cm}$, respectively. X-rays are much less sensitive to dust properties; models where X-rays dominate the excitation produce ratios of $\sim 0.07$, irrespective of the dust properties.

The $\mathrm{H}_{2}(1-0) S(1)$ and $S(2-1) S(1)$ line strengths recorded in Table 6 correspond to ratios of 0.23 and 0.3 for the SpeX and TSPEC observations; the less secure measurement of the (1-0)S(1) line suggest this ratio may best be treated as a tenuous detection which could be uncertain at the factor of two level.
Even accounting for this relatively large uncertainty, models attributing the excitation to $\mathrm{X}$-rays alone are essentially ruled out; models incorporating UV or UV + X-ray heating and dust grains $\lesssim 1 \mathrm{~mm}$ would also be difficult to reconcile with this ratio. The observed values are most consistent with, though slightly larger than, the Gredel \& Dalgarno (1995) model of shock excitation or the Nomura et al. (2007) model of UV + $\mathrm{X}$-ray irradiation of a disk with dust subject to coagulation and settling, which predict $\mathrm{H}_{2}(1-0) S(1)$ and $S(2-1) S(1)$ line ratios of 0.13 and $\sim 0.18$, respectively. Given the wealth of outflow signatures present in PTF10nvg's spectrum, we consider shocks to be the most likely source, but this conclusion is far from established.

[Fe II] is another common outflow tracer for young stars: Connelley \& Greene (2010) demonstrate that the line strengths of $\mathrm{H}_{2}$ and [Fe $\left.\mathrm{II}\right]$ are strongly correlated for a large sample of Class I protostars. The TripleSpec observation of PTF10nvg shows clear [Fe II] $\lambda 1.257$ and $\lambda 1.644 \mu \mathrm{m}$ emission; these lines may also be present in the SpeX observation, but the lower spectral resolution leads to a lower line-continuum contrast. In comparison to the Class I stars in the Connelley \& Greene (2010) sample, PTF10nvg shows relatively weak $\mathrm{H}_{2}$ and [Fe II] emission. Connelley \& Greene (2010) note, however, that the stars in their sample with weak $\mathrm{H}_{2}$ also have low levels of continuum veiling, which is not true for PTF10nvg, where we cannot detect any photospheric absorption features. 
As both the 1.257 and $1.644 \mu \mathrm{m}$ lines share the same upper level, Connelley \& Greene (2010) use their observed line fluxes to infer extinction estimates for each star in their sample, as with the $\mathrm{H}$ I-based estimates presented in Section 3.2.1. We find a line ratio in PTF10nvg of 1.48, essentially equal to that assumed by Connelley \& Greene (2010) for an unextinguished source, in strong disagreement with the extinction estimate of 6-12 which we infer from the Hi lines of PTF10nvg. Connelley \& Greene (2010) find a similar discrepancy, however, in their analysis of the Class I source $06297+1021 \mathrm{~W}$, whose H I lines imply $A_{V} \approx 18$ mag but whose [Fe II] lines suggest $A_{V} \approx-1$ mag. In many regards, $06297+1021 \mathrm{~W}$ is quite similar to PTF10nvg: in addition to their similarly incongruous $\mathrm{H}$ I and [Fe II] extinction estimates, both show weak $\mathrm{H}_{2}$ and [Fe II] emission relative to other Class I sources (Connelley \& Greene 2010 are unable to measure values for these features for $06297+1021 \mathrm{~W}$ ), both objects appear as a $K$-band point source absent significant nebulosity, and both display a rich, morphologically similar NIR emission-line spectrum.

Connelley \& Greene (2010) also explore the correlation between the strengths of the $\mathrm{H}_{2}$, [Fe II], and $\mathrm{Br}-\gamma$ emission lines: relative to their sample of Class I objects, PTF10nvg lies on the weak side of these relations, though not significantly separated from the rest of the Class I population.

\subsubsection{Optical TiO/VO Emission}

In addition to the rich atomic emission-line spectrum, the most salient feature of the optical spectrum of PTF10nvg is the broad emission that we attribute to TiO and VO. The PTF10nvg spectrum (Figure 7) looks much like an inverted mid-M spectral type star, suggesting that in addition to the hot and moderate density emission lines, there is a cooler $(\sim 1500-4000 \mathrm{~K}$, Lodders 2002; Ferguson et al. 2005; Sharp \& Burrows 2007) and high-density $\left(>10^{10} \mathrm{~cm}^{-3}\right)$ emitting component, perhaps the outer layers of a disk. Notably, the TiO emission is variable among our three spectra, being strongest just before the object brightened for the second time during the 2010 season.

To our knowledge, this is the first time such prominent hot molecular emission has been reported for a young star. There is, however, literature discussion of emission in the optical $\mathrm{TiO}$ band heads, though not the broader full bands as we observe in PTF10nvg. TiO band head emission has been mentioned in the spectra of several luminous B[e] stars (Zickgraf et al. 1989) and several red giant or supergiant dusty objects, notably VY CMa (Phillips \& Davis 1987) and U Equ (Barnbaum et al. 1996), as well as in the red nova V4332 Sgr (Goranskii \& Barsukova 2007). Most relevant to the present case is the report by Herbig (2009) that V1057 Cyg exhibited emission in 2004 in "the heads of the (0-0) bands of the $\gamma$ system of $\mathrm{TiO}$ at 7054, 7087, and $7125 \AA$ A." We have confirmed this narrow emission in our own data on V1057 Cyg but emphasize that the PTF10nvg $\mathrm{TiO}$ emission is through the full band region, not just in the band heads.

\subsubsection{Infrared $\mathrm{CO}, \mathrm{VO}$, and $\mathrm{H}_{2} \mathrm{O}$ Emission Lines}

In addition to the optical $\mathrm{TiO}$ and $\mathrm{VO}$ emission, there is also molecular emission throughout the 1-5 $\mu \mathrm{m}$ spectra of PTF10nvg. Readily apparent broad features above the likely dust-dominated red continuum are $\mathrm{VO}$ at $1.05 \mu \mathrm{m}$, several $\mathrm{H}_{2} \mathrm{O}$ bands around 1.4, 1.8, 2.0, and $2.45 \mu \mathrm{m}$ as well as the $\mathrm{Q}$ and $\mathrm{R}$ branches at 3.0 and $3.3 \mu \mathrm{m}$, and prominent $\mathrm{CO}$ first overtone band heads beginning at $2.3 \mu \mathrm{m}$. While the $\mathrm{VO}$ and $\mathrm{H}_{2} \mathrm{O}$ suggest emitting region temperatures of $<3500 \mathrm{~K}$, the $\mathrm{CO}$ emission could come from warmer $(2500-5000 \mathrm{~K})$ dense $\left(>10^{10} \mathrm{~cm}^{-3}\right)$ gas.

Connelley \& Greene (2010) display correlations between the morphology and strengths of the $\mathrm{CO}, \mathrm{Na}, \mathrm{Ca}$, and $\mathrm{Br}-\gamma$ features for Class I sources, finding that all sources with $\mathrm{CO}$ in emission also display $\mathrm{Br}-\gamma$ and $\mathrm{Na}$ in emission. PTF10nvg follows this relation and displays $\mathrm{CO}, \mathrm{Na}$, and $\mathrm{Ca}$ emission-line strengths similar to those of other Class I sources with each set of features in emission. PTF10nvg does show somewhat weaker $\mathrm{Br}-\gamma$ relative to its $\mathrm{CO}$ emission strength than most Class I sources, but is not an extreme outlier to that relation.

In contrast to the $\mathrm{CO}$ band head emission at $2.3 \mu \mathrm{m}$, the $4.7 \mu \mathrm{m} \mathrm{CO}$ fundamental band is in absorption with both the $\mathrm{P}$ and $\mathrm{R}$ branches evident.

\section{DISCUSSION}

The spectra obtained to date of PTF10nvg lack the strong FG-supergiant optical and M-supergiant NIR absorption features typical of FU Ori objects, which are interpreted as diskatmosphere dominated systems. Instead, the PTF10nvg spectra are characterized mainly by emission lines, some with kinematic signatures of formation in outflowing material. The only similarities to the FU Ori class are in the $\mathrm{Na}$ I D, K I, and He I blueshifted absorptions which are kinematically associated with strong outflows, as well as the O I $7773 \AA$ neutral velocity absorption which also may be related to winds. Despite the optical rise by over 6 mag within one year, PTF10nvg does not appear to be in the FU Ori class. This statement is based on the first year of data on this object and does not preclude that it could settle down in terms of its variability and its outflow-dominated optical and NIR spectrum to reveal FU Ori-like absorption features in the future. There is, however, a notable parallel to the FU Ori object V1057 Cyg in the He I $\lambda 10830$ profile and a similarity in the optical $\mathrm{TiO}$ emission.

The similarities of PTF10nvg to EX Lup outbursts are also slim. Neither the light curve nor the spectra are similar to this class of objects, which are interpreted as episodes of enhanced accretion but less extreme than those nominally driven by disk instability in FU Ori objects.

Instead, the PTF10nvg data bear qualitative and quantitative similarities to the 2004 outburst of V1647 Ori (also known as McNeil's Nebula), albeit with a significantly weaker wind component. Specifically, the amplitude of the optical outburst is similar, although PTF10nvg is much more unstable and photometrically variable in its outburst phase (see the $I$-band light curve given by Acosta-Pulido et al. (2007).

The PTF10nvg SpeX spectrum is a reasonable match to the one-year post-outburst spectrum of V1647 Ori in terms of the emission-line features. The most notable disagreement is the absence of broad 3.3 and $4.7 \mu \mathrm{m}$ ice features in the spectrum of PTF10nvg: those features are present at all epochs for V1647 Ori and thought to be foreground molecular cloud material not related to the outburst. The early V1647 Ori spectrum (2004 March) also showed P Cygni profiles in Paschen lines, not seen in PTF10nvg, but those profiles disappeared by 2005 March (e.g., Gibb et al. 2006). Along the same lines, compared to only $1.083 \mu \mathrm{m}$ He I visible in PTF10nvg, V1647 Ori showed strong He I absorption at both 1.083 and $2.05 \mu \mathrm{m}$ during its recent outburst, with slight red-side emission in both lines (Figure 1 of Vacca et al. 2004), but by 2004 November the $1.083 \mu \mathrm{m}$ line was much weaker, without red-side emission (Figure 1 of Gibb et al. 2006). Notably, despite the strong signatures of outflow there is 
no evidence of shocked $\mathrm{H}_{2}$ emission in V1647 Ori. PTF10nvg, in contrast, has a relatively slow wind but does possess $\mathrm{H}_{2}$ in emission. This could be a line-of-sight effect but may be an intrinsic astrophysical difference. There are several objects in the Connelley \& Greene (2010) catalog of Class I sources which show somewhat similar NIR spectra to PTF10nvg and also display $\mathrm{H}_{2}$ in emission: 16289-4449 (another eruptive variable with CO in emission), and $20453+6746$, whose spectrum is somewhat more in line with a typical Class I source but which does display some of the same emission lines in the $J$ band. $06297+1021 \mathrm{~W}$, the Class I source in the Connelley \& Greene (2010) catalog whose similarities to PTF10nvg were outlined in Section 3.2.5, does not, however, appear to show strong $\mathrm{H}_{2}$ emission.

The PTF10nvg optical spectra also appear similar to those of V1647 Ori in terms of line EWs and flux ratios, though they indicate weaker P Cygni structure in the Balmer and Ca II lines (e.g., Reipurth \& Aspin 2004a; Briceño et al. 2004), which themselves disappeared in V1647 Ori roughly one-year post-outburst (Ojha et al. 2006).

A unique feature, however, of PTF10nvg relative to any known young stellar object is the molecular $\mathrm{TiO}$ and VO seen in emission in the optical spectra. This emission has varied in strength relative to the varying continuum over a 1 month timescale. It indicates the presence of dense warm gas, perhaps the upper levels of a disk atmosphere heated by irradiation from enhanced accretion luminosity.

\section{SUMMARY}

1. We have identified PTF10nvg, a Class I protostar undergoing a strong outburst. A comparison of the source's pre- and post-outburst SED suggests that the bolometric luminosity has increased by a factor of more than 100 .

2. This outburst is presumably driven by a significant increase in PTF10nvg's mass accretion rate. We analyzed the strength of the NIR Hi lines to derive an extinction estimate of $A_{V} \sim 6-12 \mathrm{mag}$ and an accretion rate of $\dot{M} \approx$ $2.5 \times 10^{-7} M_{\odot} \mathrm{yr}^{-1}$.

3. Several wind-sensitive optical and NIR absorption lines exhibit blueshifts of several hundred $\mathrm{km} \mathrm{s}^{-1}$, indicating that PTF10nvg is driving a substantial outflow. This interpretation is supported by the presence of several spectroscopic lines thought to trace shocked gas $\left(\mathrm{H}_{2},[\mathrm{~S} \mathrm{II}]\right.$, [Fe II]).

4. Several optical $\mathrm{TiO}$ bands are seen fully in emission, a characteristic that is, to the best of our knowledge, unique to this astronomical object. This emission reveals the presence of a significant amount of dense $\left(n>10^{10} \mathrm{~cm}^{-3}\right)$, warm $(1500-4000 \mathrm{~K})$, presumably circumstellar gas.

5. During its present outburst, PTF10nvg's photometric and spectroscopic properties differ significantly from those commonly associated with EXor or FU Ori outbursts. We do identify several objects whose NIR spectra show morphological similarities to that of PTF10nvg: 06297+1021W, 16289-4449, and 20453+6746, as well as V1647 Ori, at least during its 2004-2006 outburst. Detailed monitoring of PTF10nvg, and each of these other variables, is required to ascertain the exact nature of these enigmatic sources.

The authors are grateful to Michael T. Kandrashoff, Christopher V. Griffith, A. Cucchiara, Daniel A. Perley, and A. N. Morgan for their assistance in obtaining the LRIS and Kast spectra presented here. We are also grateful to the Directors of the NASA Infrared Telescope Facility (IRTF) and
Apache Point Observatory (APO), whose allocation of Director's Discretionary Time enabled the acquisition of the SpeX and TripleSpec spectra presented here. We also thank Erika Gibb for making available archival SpeX observations of V1647 Ori, Tom Greene for a helpful discussion of NIR shock tracers, and Michael Eracleous for a useful discussion of symbiotic binaries and other accreting systems.

K.R.C. acknowledges support for this work from the Hubble Fellowship Program, provided by NASA through Hubble Fellowship grant HST-HF-51253.01-A awarded by the STScI, which is operated by the AURA, Inc., for NASA, under contract NAS 5-26555. J.S.B., D.A.P., C.K., A.A.M., and D.A.S. acknowledge support of an NSF-CDI grant, "Real-Time Classification of Massive Time-Series Data Streams" (Award 0941742). A.V.F.'s group is grateful for the support of NSF grant AST-0908886, the TABASGO Foundation, Gary and Cynthia Bengier, and the Richard and Rhoda Goldman Fund.

Some of the data presented herein were obtained at the W. M. Keck Observatory, which is operated as a scientific partnership among the California Institute of Technology, the University of California, and the National Aeronautics and Space Administration. The Observatory was made possible by the generous financial support of the W. M. Keck Foundation. The authors recognize and acknowledge the very significant cultural role and reverence that the summit of Mauna Kea has always had within the indigenous Hawaiian community. We are most fortunate to have the opportunity to conduct observations from this mountain.

PAIRITEL is operated by the Smithsonian Astrophysical Observatory (SAO) and was made possible by a grant from the Harvard University Milton Fund, a camera loan from the University of Virginia, and continued support of the SAO and UC Berkeley. The PAIRITEL project and those working on PAIRITEL data are further supported by NASA/Swift Guest Investigator Programs NNX09AQ66Q and NNX10A128G. This work was also based in part on observations obtained with the Apache Point Observatory $3.5 \mathrm{~m}$ telescope, which is owned and operated by the Astrophysical Research Consortium. We are grateful for the assistance of the staffs at all of the observatories used to obtain the data.

This research has made use of NASA's Astrophysics Data System Bibliographic Services, the SIMBAD database, operated at CDS, Strasbourg, France, the NASA/IPAC Extragalactic Database, operated by the Jet Propulsion Laboratory, California Institute of Technology, under contract with the National Aeronautics and Space Administration, and the VizieR database of astronomical catalogs (Ochsenbein et al. 2000).

This publication makes use of data products from the Two Micron All Sky Survey, which is a joint project of the University of Massachusetts and the Infrared Processing and Analysis Center/California Institute of Technology, funded by the National Aeronautics and Space Administration and the National Science Foundation.

Facilities: PO:1.2m, PO:1.5m, Hale, Keck:I, Keck:II, FLWO: $1.2 \mathrm{~m}$, ARC, Shane

\section{REFERENCES}

Acosta-Pulido, J. A., et al. 2007, AJ, 133, 2020

Antoniucci, S., Nisini, B., Giannini, T., \& Lorenzetti, D. 2008, A\&A, 479, 503

Armitage, P. J., Livio, M., \& Pringle, J. E. 2001, MNRAS, 324, 705

Aspin, C., et al. 2009, ApJ, 692, L67

Astier, P., et al. 2006, A\&A, 447, 31

Audard, M., et al. 2010, A\&A, 511, A63 
Bally, J., \& Reipurth, B. 2003, AJ, 126, 893

Barnbaum, C., Omont, A., \& Morris, M. 1996, A\&A, 310, 259

Bell, K. R., \& Lin, D. N. C. 1994, ApJ, 427, 987

Bertin, E., \& Arnouts, S. 1996, A\&AS, 117, 393

Bessell, M. S. 1999, PASP, 111, 1426

Blake, C. H., Bloom, J. S., Latham, D. W., Szentgyorgyi, A. H., Skrutskie, M. F., Falco, E. E., \& Starr, D. S. 2008, PASP, 120, 860

Bloom, J. S., Starr, D. L., Blake, C. H., Skrutskie, M. F., \& Falco, E. E. 2006 in ASP Conf. Ser. 351, Astronomical Data Analysis Software and Systems XV, ed. C. Gabriel, C. Arviset, D. Ponz, \& S. Enrique (San Francisco, CA: ASP), 751

Bloom, J. S., et al. 2009, ApJ, 691, 723

Bochanski, J. J., West, A. A., Hawley, S. L., \& Covey, K. R. 2007, AJ, 133, 531

Boley, A. C., Mejía, A. C., Durisen, R. H., Cai, K., Pickett, M. K., \& D’Alessio, P. 2006, ApJ, 651, 517

Briceño, C., et al. 2004, ApJ, 606, L123

Burrows, D. N., et al. 2005, Space Sci. Rev., 120, 165

Butler, N. R., \& Kocevski, D. 2007, ApJ, 668, 400

Carpenter, J. M. 2001, AJ, 121, 2851

Cenko, S. B., et al. 2006, PASP, 118, 1396

Connelley, M. S., \& Greene, T. P. 2010, AJ, 140, 1214

Covey, K., et al. 2007, AJ, 134, 2398

Cushing, M. C., Vacca, W. D., \& Rayner, J. T. 2004, PASP, 116, 362

Dobashi, K., Bernard, J.-P., Yonekura, Y., \& Fukui, Y. 1994, ApJS, 95, 419

Edwards, S., Fischer, W., Hillenbrand, L., \& Kwan, J. 2006, ApJ, 646, 319

Edwards, S., Fischer, W., Kwan, J., Hillenbrand, L., \& Dupree, A. K. 2003, ApJ, 599, L41

Egan, M. P., Price, S. D., Shipman, R. F., Gugliotti, G. M., Tedesco, E. F., Moshir, M., \& Cohen, M. 1999, in ASP Conf. Ser. 177, Astrophysics with Infrared Surveys: A Prelude to SIRTF, ed. Michael D. Bicay, Roc M. Cutri, \& Barry F. Madore (San Francisco, CA: ASP), 404

Enoch, M. L., Evans, N. J., Sargent, A. I., \& Glenn, J. 2009, ApJ, 692, 973

Fedele, D., van den Ancker, M. E., Petr-Gotzens, M. G., \& Rafanelli, P. 2007, A\&A, 472, 207

Ferguson, J. W., Alexander, D. R., Allard, F., Barman, T., Bodnarik, J. G., Hauschildt, P. H., Heffner-Wong, A., \& Tamanai, A. 2005, ApJ, 623, 585

Finkbeiner, D. P., et al. 2004, AJ, 128, 2577

Fischer, W., Kwan, J., Edwards, S., \& Hillenbrand, L. 2008, ApJ, 687, 1117

Gibb, E. L., Rettig, T. W., Brittain, S. D., Wasikowski, D., Simon, T., Vacca, W. D., Cushing, M. C., \& Kulesa, C. 2006, ApJ, 641, 383

Goranskii, V. P., \& Barsukova, E. A. 2007, Astron. Rep., 51, 126

Gredel, R., \& Dalgarno, A. 1995, ApJ, 446, 852

Greene, T. P., Aspin, C., \& Reipurth, B. 2008, AJ, 135, 1421

Guieu, S., et al. 2009, ApJ, 697, 787

Hamann, F., \& Persson, S. E. 1992, ApJS, 82, 247

Hartigan, P., Edwards, S., \& Ghandour, L. 1995, ApJ, 452, 736

Hartmann, L., \& Kenyon, S. J. 1996, ARA\&A, 34, 207

Herbig, G. H. 1958, ApJ, 128, 259

Herbig, G. H. 1989, in European Southern Observatory Conf. Workshop Proc., Vol. 33, ed. B. Reipurth (Garching: ESO), 233

Herbig, G. H. 2008, AJ, 135, 637

Herbig, G. H. 2009, AJ, 138, 448

Herbig, G. H., Aspin, C., Gilmore, A. C., Imhoff, C. L., \& Jones, A. F. 2001, PASP, 113, 1547

Horne, K. 1986, PASP, 98, 609

Ishihara, D., et al. 2010, A\&A, 514, A1

Joy, A. H. 1945, ApJ, 102, 168

Kastner, J. H., et al. 2006, ApJ, 648, L43

Kley, W., \& Lin, D. N. C. 1999, ApJ, 518, 833

Kwan, J., Edwards, S., \& Fischer, W. 2007, ApJ, 657, 897

Lada, C. J. 1987, in Proc. Symp. on Star Forming Regions, Vol. 115, ed. M. Peimbert \& J. Jugaku (Dordrecht: Reidel), 1
Law, N. M., et al. 2009, PASP, 121, 1395

Lodato, G., \& Clarke, C. J. 2004, MNRAS, 353, 841

Lodders, K. 2002, ApJ, 577, 974

Lorenzetti, D., Giannini, T., Larionov, V. M., Kopatskaya, E., Arkharov, A. A., De Luca, M., \& Di Paola, A. 2007, ApJ, 665, 1182

Lorenzetti, D., Larionov, V. M., Giannini, T., Arkharov, A. A., Antoniucci, S., Nisini, B., \& Di Paola, A. 2009, ApJ, 693, 1056

Magnier, E. A., Volp, A. W., Laan, K., van den Ancker, M. E., \& Waters, L. B. F. M. 1999, A\&A, 352, 228

Matheson, T., Filippenko, A. V., Ho, L. C., Barth, A. J., \& Leonard, D. C. 2000, AJ, 120, 1499

McCarthy, J. K., et al. 1998, Proc. SPIE, 3355, 81

McNeil, J. W., Reipurth, B., \& Meech, K. 2004, IAU Circ., 8284, 1

Meyer, M. R., Calvet, N., \& Hillenbrand, L. A. 1997, AJ, 114, 288

Miller, J. S., \& Stone, R. P. S. 1993, Lick Obs. Tech. Rep. 66 (Santa Cruz, CA: Lick Obs.)

Monet, D. G., et al. 2003, AJ, 125, 984

Muzerolle, J., Hartmann, L., \& Calvet, N. 1998, AJ, 116, 2965

Nisini, B., Antoniucci, S., Giannini, T., \& Lorenzetti, D. 2005, A\&A, 429, 543

Nomura, H., Aikawa, Y., Tsujimoto, M., Nakagawa, Y., \& Millar, T. J. 2007, ApJ, 661,334

Ochsenbein, F., Bauer, P., \& Marcout, J. 2000, A\&AS, 143, 23

Odenwald, S. F. 1989, AJ, 97, 801

Ogura, K., Sugitani, K., \& Pickles, A. 2002, AJ, 123, 2597

Ojha, D. K., et al. 2006, MNRAS, 368, 825

Oke, J. B., \& Gunn, J. E. 1982, PASP, 94, 586

Oke, J. B., et al. 1995, PASP, 107, 375

Peneva, S. P., Semkov, E. H., Munari, U., \& Birkle, K. 2010, A\&A, 515, A24

Perley, D. A., et al. 2010, MNRAS, 406, 2473

Phillips, J. G., \& Davis, S. P. 1987, PASP, 99, 839

Rau, A., et al. 2009, PASP, 121, 1334

Rayner, J. T., Toomey, D. W., Onaka, P. M., Denault, A. J., Stahlberger, W. E., Vacca, W. D., Cushing, M. C., \& Wang, S. 2003, PASP, 115, 362

Rebull, L. M., et al. 2010, ApJS, 186, 259

Reipurth, B., \& Aspin, C. 2004a, ApJ, 606, L119

Reipurth, B., \& Aspin, C. 2004b, ApJ, 608, L65

Reipurth, B., Aspin, C., Beck, T., Brogan, C., Connelley, M. S., \& Herbig, G. H. 2007, AJ, 133, 1000

Sharon, C., Hillenbrand, L., Fischer, W., \& Edwards, S. 2010, AJ, 139, 646

Sharp, C. M., \& Burrows, A. 2007, ApJS, 168, 140

Skrutskie, M. F., et al. 2006, AJ, 131, 1163

Steidel, C. C., Shapley, A. E., Pettini, M., Adelberger, K. L., Erb, D. K., Reddy, N. A., \& Hunt, M. P. 2004, ApJ, 604, 534

Szeifert, T., Hubrig, S., Schöller, M., Schütz, O., Stelzer, B., \& Mikulášek, Z. 2010, A\&A, 509, L7

Vacca, W. D., Cushing, M. C., \& Rayner, J. T. 2003, PASP, 115, 389

Vacca, W. D., Cushing, M. C., \& Simon, T. 2004, ApJ, 609, L29

van Dokkum, P. G. 2001, PASP, 113, 1420

Voges, W., et al. 1999, A\&A, 349, 389

Vorobyov, E. I., \& Basu, S. 2005, ApJ, 633, L137

Wade, R. A., \& Horne, K. 1988, ApJ, 324, 411

Walter, F. M., Stringfellow, G. S., Sherry, W. H., \& Field-Pollatou, A. 2004, AJ, 128,1872

Welin, G. 1973, A\&AS, 9, 183

Whelan, E. T., et al. 2010, ApJ, 720, L119

White, R. J., \& Hillenbrand, L. A. 2004, ApJ, 616, 998

Wilson, J. C., et al. 2004, Proc. SPIE, 5492, 1295

Zacharias, N., Monet, D. G., Levine, S. E., Urban, S. E., Gaume, R., \& Wycoff, G. L. 2005, VizieR Online Data Catalog, 1297, 0

Zhu, Z., Hartmann, L., Gammie, C., \& McKinney, J. C. 2009, ApJ, 701, 620

Zickgraf, F., Wolf, B., Stahl, O., \& Humphreys, R. M. 1989, A\&A, 220, 206 